\title{
ÉTUDE D'UN ÉCOULEMENT \\ MIXTE AIR-EAU VERTICAL DESCENDANT: \\ RÉGIMES, ÉVOLUTION DE LA \\ CONCENTRATION
}

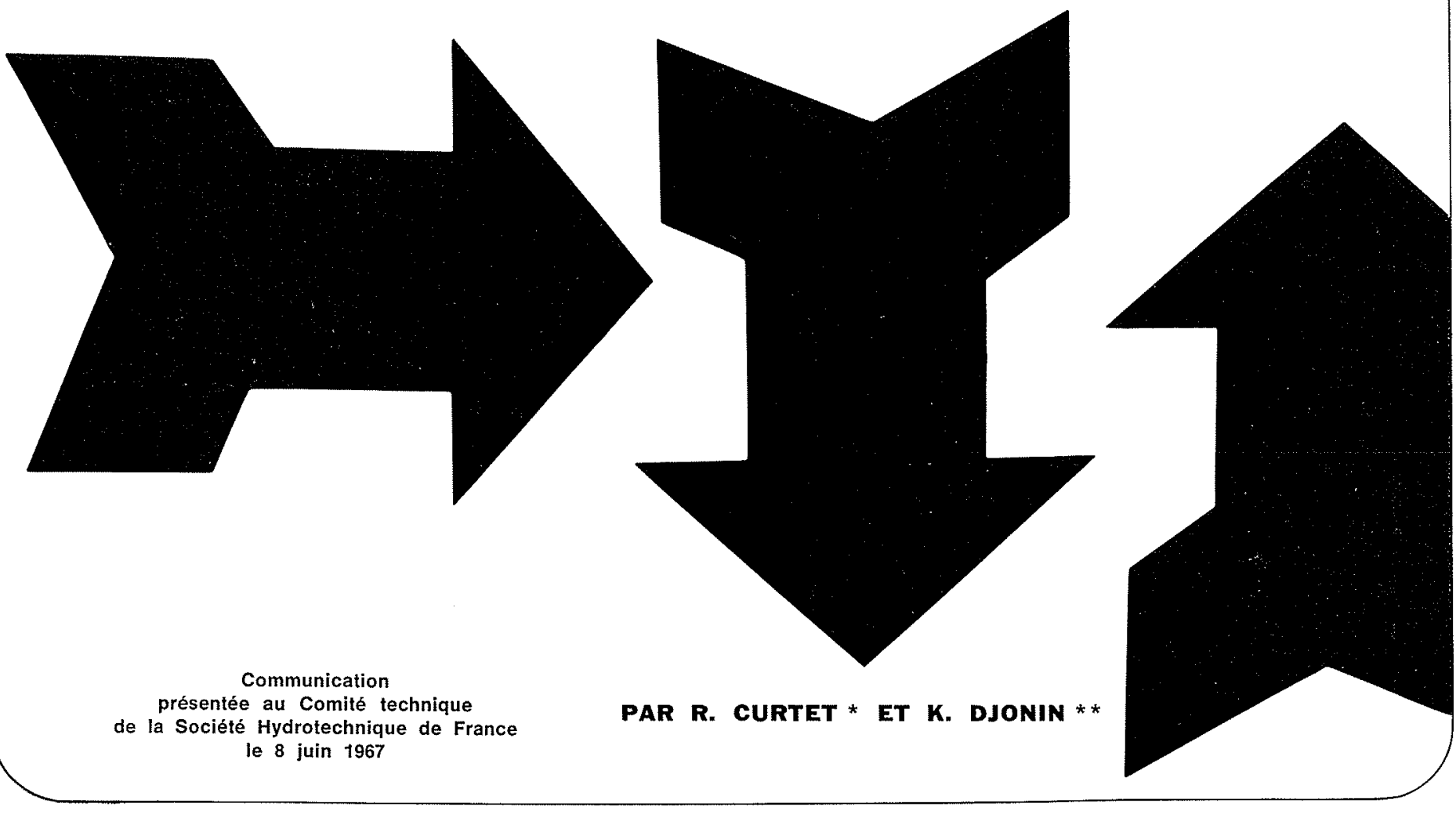

Les écoulements biphasés ont la fâcheuse réputation d'être beaucoup plus complexes que les écoulements monophasés. Nous nous proposons de montrer sur un exemple comment il est possible, malgré cette apparente complexité, de classer, d'analyser, et même de prévoir dans une certaine mesure les différentes structures observées.

Ayant en vue les idées directrices, nous ne citerons ici qu'une faible partie des résultats d'essais inclus dans le mémoire original [1].

\section{I. - Etude générale}

\section{A - Dispositif expérimental. Notations.}

Les figures $1 a$ et $b$ donnent le schéma du montage utilisé ainsi que deux des aspects de l'émulsion air-eau engendrée par un jet d'eau issu d'un diaphragme placé dans une conduite verticale. Les éléments essentiels de l'installation, que l'on retrouve sur la figure 2 , sont un réservoir $R_{0}$, un tronçon de conduite $T_{2}$ branché sur le réservoir $R_{0}$ (section $\mathrm{S}_{0}$, diamètre $\left.\mathrm{D}_{0}\right)$, un diaphragme $\left(s_{0}, d_{0}\right)$, la conduite principale $C(S, D)$ en plexiglas, située en aval du diaphragme; à l'extrémité aval de $\mathrm{C}$, est placée une vanne-pointeau $R_{1}$ à réglage fin. A la sortie de $R_{1}$, à l'endroit où les filets liquides retrouvent leur parallélisme, nous convenons de définir le plan horizontal de référence des cotes et des

* Maître de Recherches au C.N.R.S.

* Docteur-Ingénieur, Ingénieur au Laboratoire d'Avala (Yongoslavie). charges, $\mathrm{H}$ étant la charge totale (cote du plan d'eau dans le réservoir $R_{0}$ ).

Le jet d'eau sort du diaphragme de diamètre $d_{0}$; les filets liquides sont sensiblement parallèles dans la section $s$ (diamètre $d$ ), mais le diamètre du jet continue à décroître jusqu'en $d_{i}$ dans la section d'impact $s_{i}$. A la sortie de la vanne $R_{1}$, on peut définir également une section contractée $\sigma$. Notons à ce propos qu'il suffit, pour le but de l'étude actuelle, de caractériser la vanne $R_{1}$ par une certaine ouverture qui sera justement la section $\sigma$.

Soit $\mathrm{H}^{\prime}$ la hauteur de la face aval du diaphragme au-dessus du plan de référence, et $H_{1}$ définie par $\mathrm{H}_{1}=\mathrm{H}-\mathrm{H}^{\prime}$. Une prise de pression statique placée un peu en amont de la vanne $R_{1}$ indique une hauteur d'eau $\mathrm{H}_{\mathrm{S}}$ au-dessus du plan de référence; appelons $\Delta H$ la différence de cote entre la surface libre de l'eau dans la conduite et le niveau dans le manomètre.

Le diamètre $\mathrm{D}$ de la conduite $\mathrm{C}$ étant demeuré invariable ( $\mathrm{D}=46,4 \mathrm{~mm}, \mathrm{~S}=16,9 \mathrm{~cm}^{2}$ ) nous avons mis en place successivement différents diaphragmes $\left(d_{0}=4,6,8,10,12,14,16,18,20,22,25,29\right.$ et $34 \mathrm{~mm}$ ). Pour chacun d'eux, nous avons étudié divers débits d'eau $Q_{e}$, obtenus par l'intermédiaire du réservoir $R_{0}$ sous pression raccordé à la cheminée $\mathrm{T}_{4}$ munie d'un déversoir $\mathrm{D}_{1}$ réglable. Lors de chaque essai, $d_{0}$ et $Q_{e}$ étant imposés, nous avons fait varier la longueur d'impact $\mathrm{L}$ du jet à l'aide de la vanne $R_{1}$ et effectué pour chaque valeur de $L$ une étude qualitative, puis quantitative, de l'écoulement mixte dans la conduite $\mathrm{C}$. 

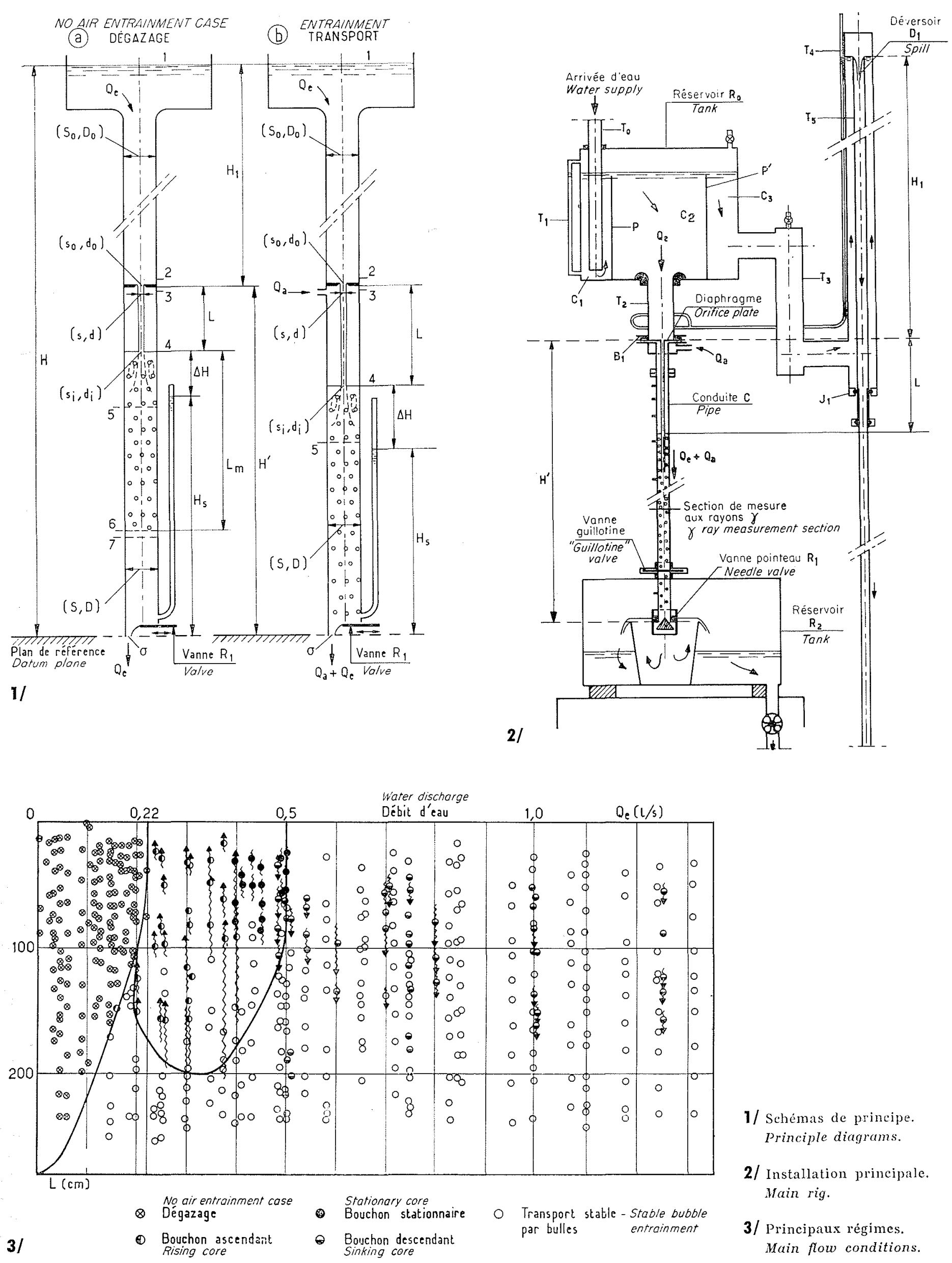

1/ Schémas de prineipe. Principle diagrams.

2/ Installation principale. Main rig.

3/ Principanx régimes. Main flow conditions. 


\section{B - Principaux régimes observés.}

Si l'on fait croître la longueur de chute libre L du jet, on constate que, dans une première phase, I'émulsion s'arrête à une certaine hauteur dans la conduite (fig. 1 a). Ce régime, que nous appelons « dégazage », correspond à un débit d'air $Q_{a}$ nul à travers la conduite $C$; il se caractérise par une longueur d'émulsion $L_{m}$ de la colonne. Selon les valeurs de $\mathrm{Q}_{\theta}$ et $\mathrm{L}$, on note les deux aspects du front de discontinuité à la base de l'émulsion, que l'on voit sur les photos $4 a$ et $4 b$ : présence ou non de petites bulles dans le liquide au-dessous du front, mais dans les deux cas, changement très net de structure de l'émulsion.

Au fur et à mesure que nous augmentons la longueur d'impact $L$, en ouvrant la vanne $R_{1}$, la base de la colonne émulsionnée descend; lorsqu'elle atteint la vanne, le débit d'air cesse d'être nul. Audelà de cette limite, apparaît le régime de «transport ». L'expérience montre que la limite entre le dégazage et le transport est pratiquement indépendante du diaphragme utilisé. Par contre, elle dépend du débit d'eau $\mathrm{Q}_{e}$ et de la longueur de chute libre L, comme le montre la figure 3 .

Le transport se présente habituellement sous l'aspect d'une émulsion de bulles à peu près homogènes, se déplaçant de haut en bas dans la conduite en un mouvement relativement régulier malgré la turbulence. Nous l'appelons transport stable, par opposition avec le transport comportant des bouchons instables, qui se forment dans certaines conditions, et de la manière décrite sur les photos $5 a, b$ et $c$ : dans une région bien définie près de l'axe de la conduite (si $d_{0}, Q_{c}$ et $\mathrm{L}$ sont donnés) il apparaît une coalescence qui se développe de proche en proche et conduit à la formation d'un bouchon d'air se propageant à travers l'émulsion homogène: ce bouchon peut-être ascendant, stationnaire, ou descendant, selon les cas.
La figure 3, où ont été regroupés un grand nombre d'essais correspondant à divers diaphragmes, met en évidence plusieurs faits remarquables. Lorsque le débit d'eau $Q_{c}$ dépasse la valeur $0,22 \mathrm{l} / \mathrm{s}$, il n'est plus possible d'observer le régime de dégazage. Un bouchon ascendant ne peut exister que si le débit d'eau est compris entre 0,2 et $0,4 \mathrm{l} / \mathrm{s}$ et un bouchon stationnaire seulement si le débit d'eau est entre 0,4 et $0,5 \mathrm{l} / \mathrm{s}$. Au-delà de $0,5 \mathrm{l} / \mathrm{s}$, s'il apparaît un bouchon, celui-ci ne peut être que descendant. On voit ainsi mis en évidence le rôle essentiel joué par le débit d'eau sur la possibilité d'existence des différents régimes.

\section{C - Dispositifs de mesure.}

L'installation représentée sur la figure 2 comporte un réservoir $R_{2}$ utilisé pour la mesure par capacité du débit d'eau; un compteur volumétrique de précision a permis la détermination du débit d'air $Q_{a}$ (transport) ou du volume d'air $V_{u}$ introduit dans la conduite $\mathrm{C}$ (dégazage). Rappelons à ce propos que la concentration de transport $c_{t}$ est définie par le rapport $\mathrm{Q}_{a} /\left(\mathrm{Q}_{a}+\mathrm{Q}_{e}\right)$.

Le long du tube $\mathrm{C}$, sont placées un certain nombre de prises de pression statique reliées à des tubes manométriques à eau.

La concentration moyenne spatiale $c_{s,}$ d'air dans l'eau à travers la colonne en émulsion a été déterminée par une méthode de «hold-up », à l'aide d'une vanne-guillotine (fig. 2) et d'un déflecteur de jet.

Enfin, à une distance de la base du diaphragme égale à $2,22 \mathrm{~m}$, est placé le dispositif de mesure de concentration spatiale par rayons gamma, décrit sur les figures 6 . Une source au caesium 137 (fig. 6 a) émet un faisceau de rayons gamma diaphragmé par deux collimateurs au plomb situés de part et d'autre du conduit de mesure. Les impulsions dues au rayonnement sont détectées par une

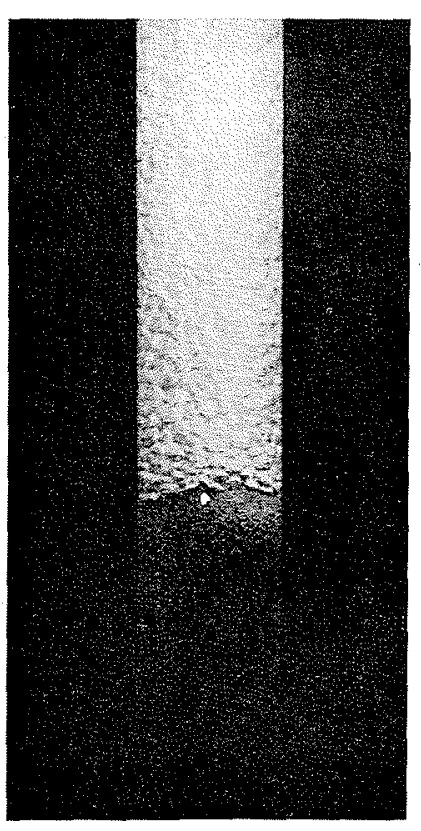

4 a ef $b /$

(a)

Extrémité aval de l'émulsion (Dégazage).

Downstream end of emulsion (no air entrainment).

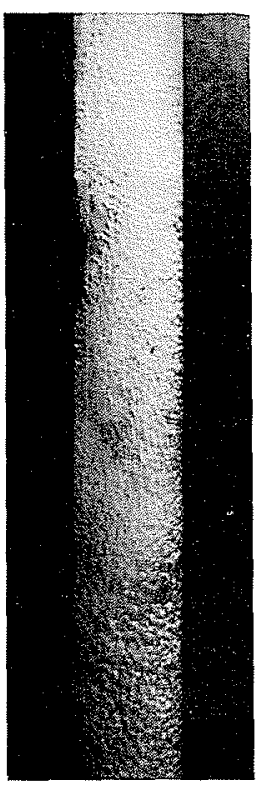

(a)

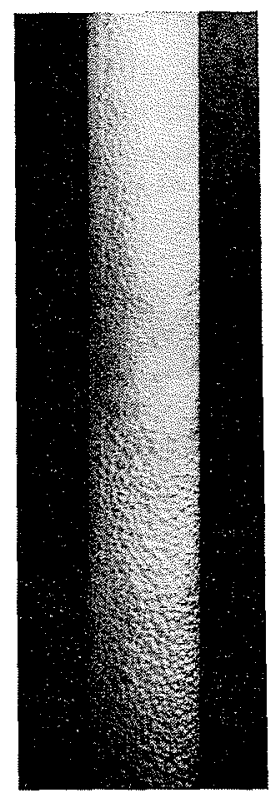

(b)

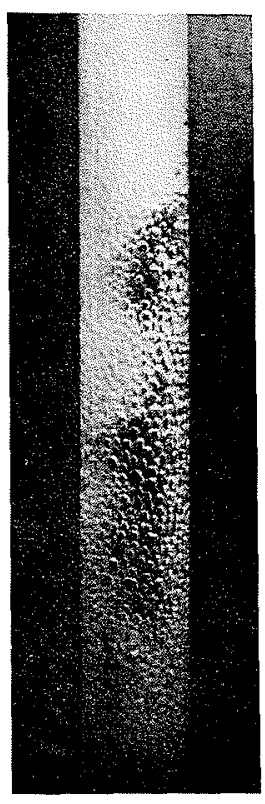

(c)
$5 a, b, c /$

Apparition d'une poche d'air dans une émulsion homogène. Occurrence of an air pocket in a homogeneous emulsion. 


\section{R. CURTET et K. DJONIN}

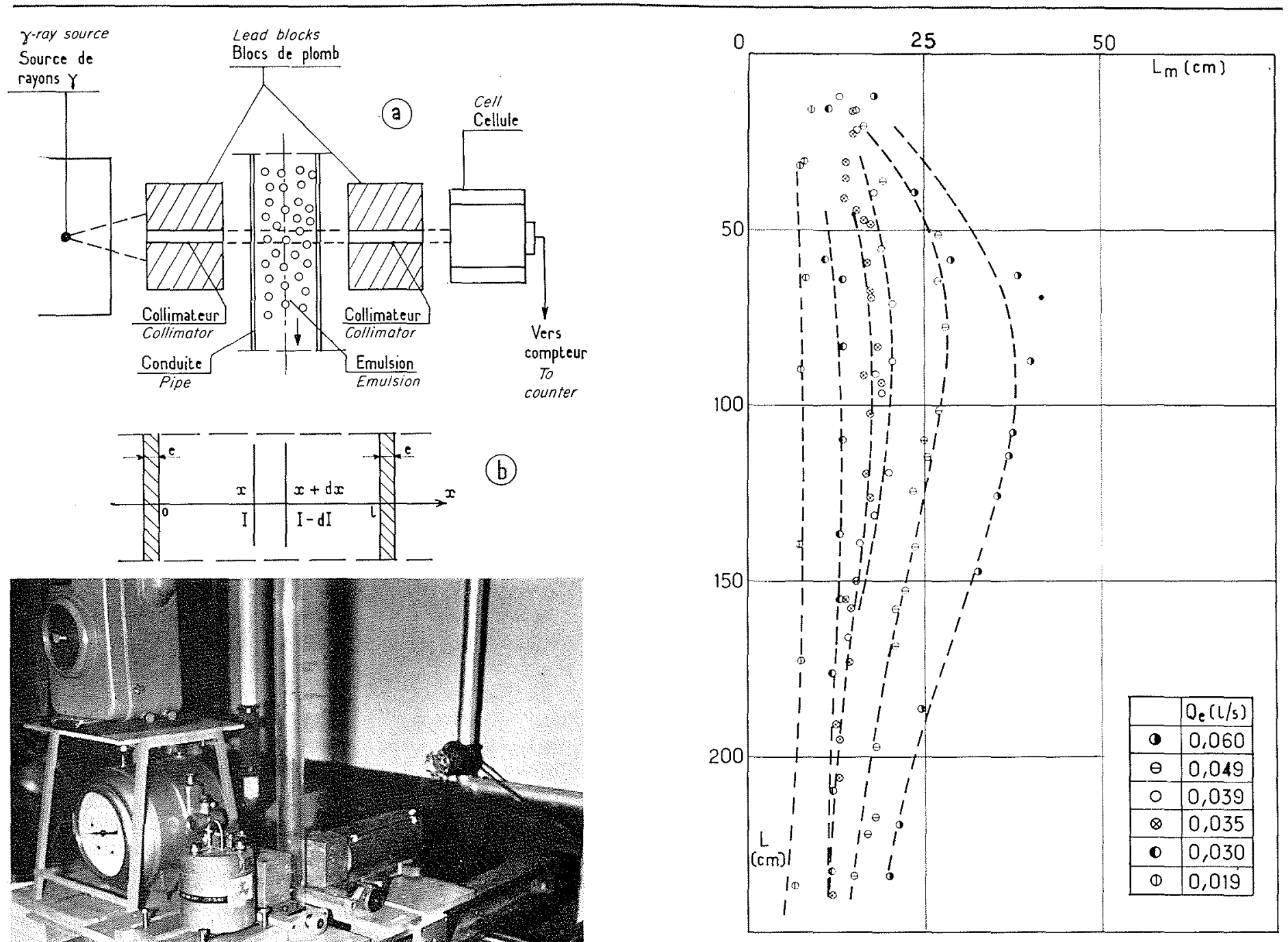

8 a/ Dégazage. Longueur d'émulsion $\left(d_{0}=4 \mathrm{~mm}\right)$. No air entrainment case. Length of emulsion $\left(\mathrm{d}_{o}=4 \mathrm{~mm}\right)$.

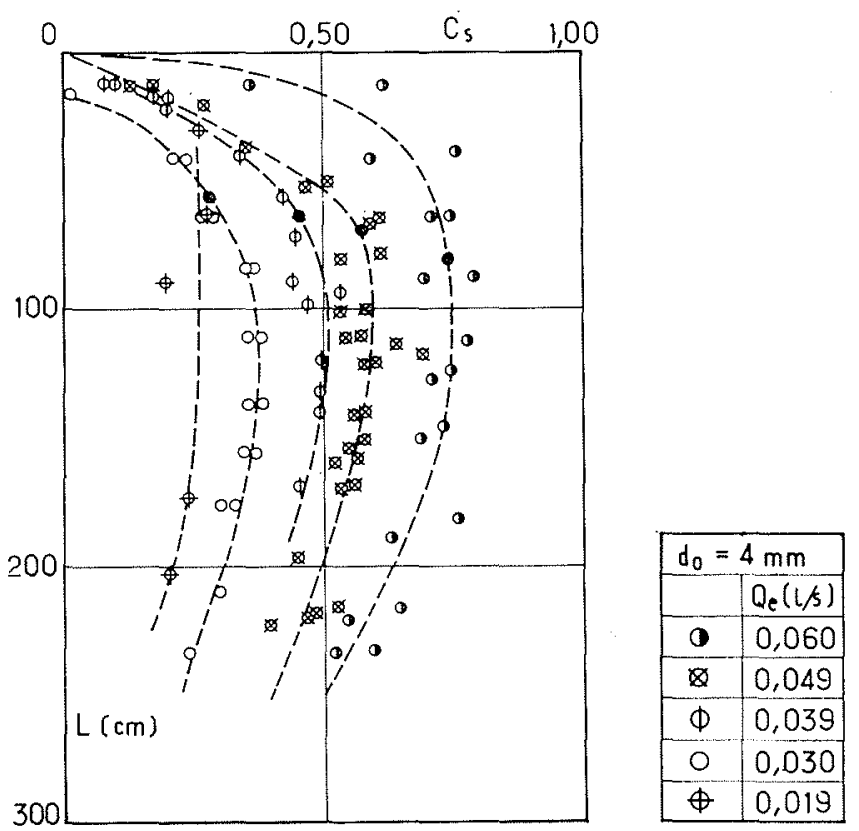

7/ Dégazage. Courbes expérimentales. No air entrainment case. Experimental curves.

$8 \mathrm{~b} /$ Dégazage. Concentration moyenne spatiale. No air entrainment case. Mean space concentration. 
9 a/ Dégazage. Longueur maximale d'émulsion. No air entrainment case. Maximum emulsion length.

9 b/ Dégazage. Concentration maximale. No air entrainment case. Maximum concentration.
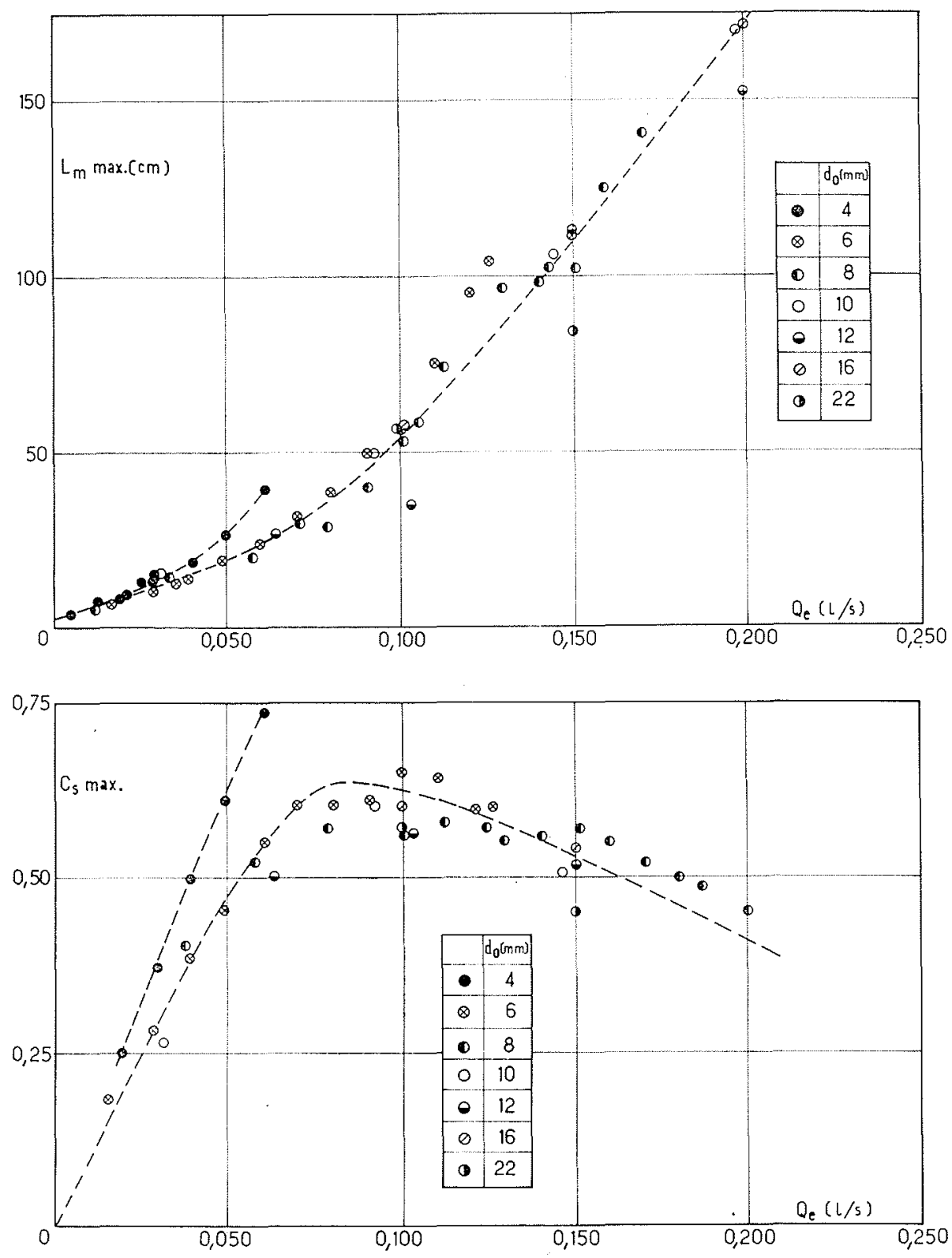

cellule et dénombrées par un compteur. Le schéma $b$ rappelle que l'intensité I du rayonnement décroît sous l'effet de l'absorption par le milieu; les calculs conduisent à une relation logarithmique entre la concentration moyenne d'air dans l'eau comprise dans le faisceau et le nombre d'impulsions reçues par la cellule. L'ensemble du dispositif de mesure est monté sur un chariot, commandé par vis micrométrique (photo $6 \mathrm{c}$ ) et permettant une étude de la répartition de concentration en une section du conduit. Notons à ce propos que la mesure aux rayons gamma ne donne pas une valeur locale, mais une moyenne de la concentration le long d'une corde. Nous verrons plus loin comment passer de l'une à l'autre.

\section{II. - Dégazage}

Pour étudier le dégazage, nous avons introduit dans la conduite $\mathrm{C}$ un volume $\mathrm{V}_{a}$ connu d'air (à la pression atmosphérique), pour $d_{0}$ et $Q_{0}$ constants, et chaque fois nous avons noté la longueur d'impact $L$, d'émulsion $L_{m}$ et la différence de niveau $\Delta H$. Nous avons ainsi obtenu un ensemble de courbes analogues à celles portées sur la figure $7: \mathrm{AB}=\mathrm{L}$, $\mathrm{BD}=\mathrm{L}_{m}, \mathrm{BE}=\Delta \mathrm{H}, \quad \mathrm{AC}=\mathrm{L}_{a}=\mathrm{V}_{u} / \mathrm{S}, \mathrm{S}$ étant la section du conduit. De la figure, on déduit immédiatement la concentration spatiale moyenne $c_{s}$, égale au rapport du volume d'air au volume total d'émulsion :

$$
c_{s}=\mathrm{BC} / \mathrm{BD}
$$

\section{A - Evolution de $L_{m}$ et de $c_{s}$.}

Sur les figures 8 , nous donnons à titre d'exemple quelques courbes montrant l'évolution en fonction de la longueur de chute libre $L$, pour différents débits d'eau et pour le diaphragme de $4 \mathrm{~mm}$ de diamètre, de la longueur d'émulsion $\mathrm{L}_{m}$ (fig. $8 a$ ) et de la concentration spatiale moyenne $c_{s}$ (fig. $8 \mathrm{~b}$ ). On constate notamment que chaque courbe présente un maximum de $\mathrm{L}_{m}$ ou de $c_{s}$. Sur les figures $9 a$ et $b$; 
sont reportées les valeurs maximales ainsi obtenues, en fonction du débit d'eau, pour différents orifices. Si l'on met à part les points relatifs au diaphragme de $4 \mathrm{~mm}$, les autres points paraissent se rassembler autour d'une courbe unique, indépendante du diamètre de l'ajutage.

\section{B - Equations générales.}

La question se pose de savoir s'il est possible, à l'aide des seules équations générales du mouvement, de prévoir les propriétés globales de l'émulsion, et notamment $\mathrm{L}_{m}$ et $c_{s}$, en fonction des données de l'installation ( $\left.\mathrm{S}, \mathrm{H}, \mathrm{Q}_{e}, d_{0}, \sigma\right)$. Un calcul élémentaire, basé sur l'application de la relation de Bernoulli et du théorème des quantités de mouvement, peut être effectué dans les hypothèses suivantes de première approximation :

- répartition des vitesses uniforme en une section;

- pertes de charge par frottement négligées le long de la conduite et dans l'émulsion, les vitesses d'eau étant, dans le cas du dégazage, inférieures à $0,15 \mathrm{~m} / \mathrm{s}$.

-- émulsion homogène de concentration spatiale $c_{s}$

Le calcul conduit à la relation :

$$
\frac{\mathrm{Q}_{c}}{2 \mathrm{gHS}}==\frac{1-\left(\mathrm{V}_{a} / \mathrm{HS}\right)}{\left[\left(\mathrm{S} / s_{i}\right)-1\right]^{2}+\left(\mathrm{S}^{2} / \sigma^{2}\right)}
$$

et prend en compte la récupération partielle de pression entre les sections 4 et 5 (fig. $1 a$ ), du fait de l'élargissement brusque.

Si le terme $\mathrm{S} / s_{i}$ est grand devant l'unité, l'équation (1) se réduit à l'équation (2) :

$$
\mathrm{H}-\mathrm{H}_{1}=\mathrm{H}_{\mathrm{S}}+\frac{\mathrm{V}_{a}}{\mathrm{~S}}=\mathrm{H}_{\mathrm{S}}+\Delta \mathrm{H}+\mathrm{L}
$$

En d'autres termes, la perte de charge $\Delta H$ à travers l'émulsion représente essentiellement la variation de densité de l'émulsion : elle n'en diffère que par la récupération éventuelle de pression étoilée due à l'élargissement brusque.

L'expérience montre que, l'installation étant en place, si l'on se donne le diamètre $d_{0}$ de l'ajutage, le débit d'eau $Q_{c}$ et une ouverture $\sigma$ de la vanne $R_{1}$, tout est fixé, notamment le volume d'air $V_{a}$ présent dans la conduite, la concentration spatiale $c_{s}$ et la longueur d'émulsion $\mathrm{L}_{m}$. Que donnent sur ce point les équations générales? Prenons par exemple la forme (2), particulièrement simple: $\mathrm{H}$ et $\mathrm{H}_{1}$ sont des données, ainsi que $\mathrm{H}_{\mathrm{S}}$ puisque le débit $\mathrm{Q}_{c}$ et l'ouverture $\sigma$ sont imposés. Par conséquent, l'équation (2) permet d'en déduire le volume d'air $V_{a}$. Mais on ne peut connaître ni la longueur d'émulsion $\mathrm{L}_{m}$, ni la concentration $c_{s}$; on sait simplement que:

$$
c_{s}=\Delta \mathrm{H} / \mathrm{L}_{m} \quad \text { et que } \quad V_{a} / \mathrm{S}=\mathrm{L}+\Delta \mathrm{H}
$$

\section{C - Recherche d'autres relations.}

Nous avons essayé de trouver d'autres relations entre les variables. C'est ainsi que nous avons obtenu le groupement des points expérimentaux indiqué sur la figure 10 : en ordonnées, est portée la longueur d'émulsion $\mathrm{L}_{m}$, et en abscisses, la valeur absolue de la vitesse moyenne de glissement définie par :

$$
\mathrm{V}_{g}=-\mathrm{Q}_{\mathrm{c}} / \mathrm{S}\left(1-c_{s}\right)
$$

En fait, si la variable $g \mathrm{~L}_{m}$ ne dépendait que de la vitesse de glissement, l'analyse dimensionnelle conduirait immédiatement à un nombre de Froude caractéristique $\mathscr{g}$ tel que :

$$
\mathscr{9}=\mathrm{V}_{g} / \sqrt{g \mathrm{~L}_{\mathrm{m}}}=\mathrm{Cte}
$$

Nous avons tracé sur la figure 10 la parabole :

$$
g \mathrm{~L}_{m}=265 \mathrm{~V}_{g^{2}} \quad \text { soit } \quad \mathscr{F}=0,06
$$

Pour les valeurs de $\mathrm{L}_{m}$ supérieures à $25 \mathrm{~cm}$, cette parabole passe convenablement à travers les points expérimentaux. Les valeurs de $\mathrm{L}_{n}$ inférieures à $25 \mathrm{~cm}$ sont relatives à la région de recirculation, et le régime de l'émulsion n'est pas dynamiquement établi.

Admettons, pour simplifier le raisonnement, que nous connaissions une relation entre la vitesse de glissement $V_{g}$ et la longueur $\mathbf{L}_{m}$. Si le débit $\mathrm{Q}$. est connu (ainsi que S), on déduit de (3) une relation entre $L_{m}$ et $c_{s}$, et comme $c_{s}=\Delta H / L_{n}$ en première approximation, une relation également entre $\Delta \mathrm{H}$ et $\mathrm{L}_{m}$. Mais nous ne sommes pas encore au bout de nos peines puisque, dans l'équation: $\mathrm{V}_{a} / \mathrm{S}=\mathrm{L}+\Delta \mathrm{H}$ seul le volume $\mathrm{V}_{a}$ est connu. Il reste encore à trouver une relation entre les variables, par exemple entre la concentration et le débit d'eau; nous reverrons ce point au chapitre $V$.

\section{III. - Transport d'air stable}

Comme pour le dégazage, les trois paramètres essentiels sont le débit d'eau $\mathrm{Q}_{e}$, le diamètre $d_{0} \mathrm{du}$ diaphragme, et la longueur de chute libre $\mathrm{L}$ du jet.

La figure 11 donne un exemple d'évolution du débit d'air $Q_{a}$ en fonction de $\mathrm{L}$, pour différentes valeurs de $Q_{e}$ et pour $d_{0}=14 \mathrm{~mm}$. La figure 12 met en évidence, pour une longueur $\mathrm{L}$ constante et égale à $150 \mathrm{~cm}$, la variation de $Q_{a}$ en fonction de $Q_{e}$ et de $d_{0}$.

\section{A - Courbes de pression et perte de charge à travers l'émulsion.}

Sur la figure 13, nous montrons quelques courbes de répartition de la pression statique le long de la conduite, $c_{t}$ étant la concentration de transport. Chaque courbe comprend plusieurs parties: une région $\mathrm{OA}$ (droite à $45^{\circ}$ ) correspondant à la pression atmosphérique régnant autour du jet tombant en chute libre; de $A$ à $B$, récupération de pression dans l'élargissement brusque (soit de 4 à 5 sur la figure $1 b$ ); variation linéaire le long de $\mathrm{BCD}$, due à l'émulsion en régime établi. La pente $i$ de cette droite dépend principalement de la variation de densité due à la concentration en air et également de la perte de charge par frottement au sein de l'émulsion. Une analyse des résultats nous a permis 


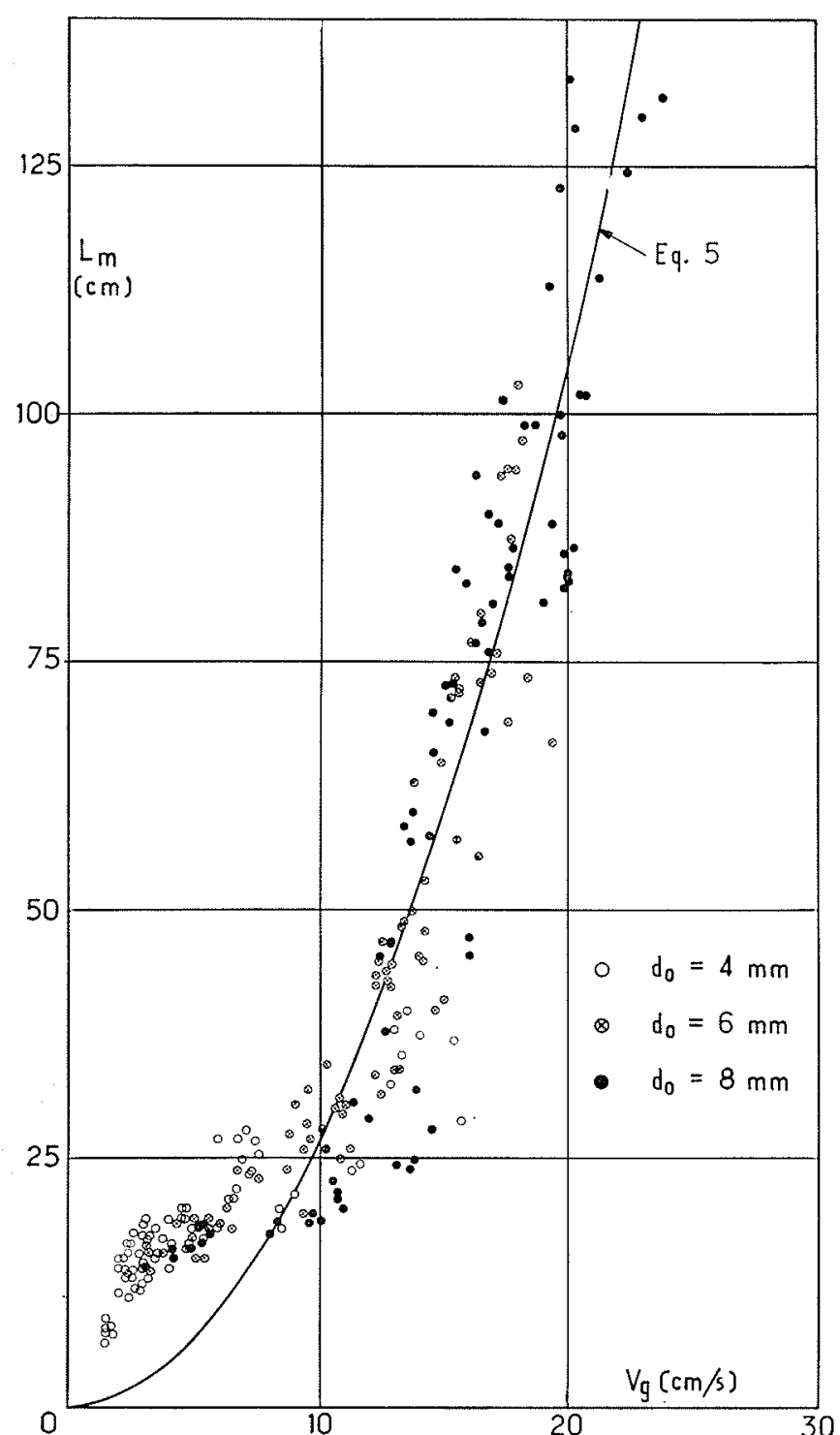

$10 /$

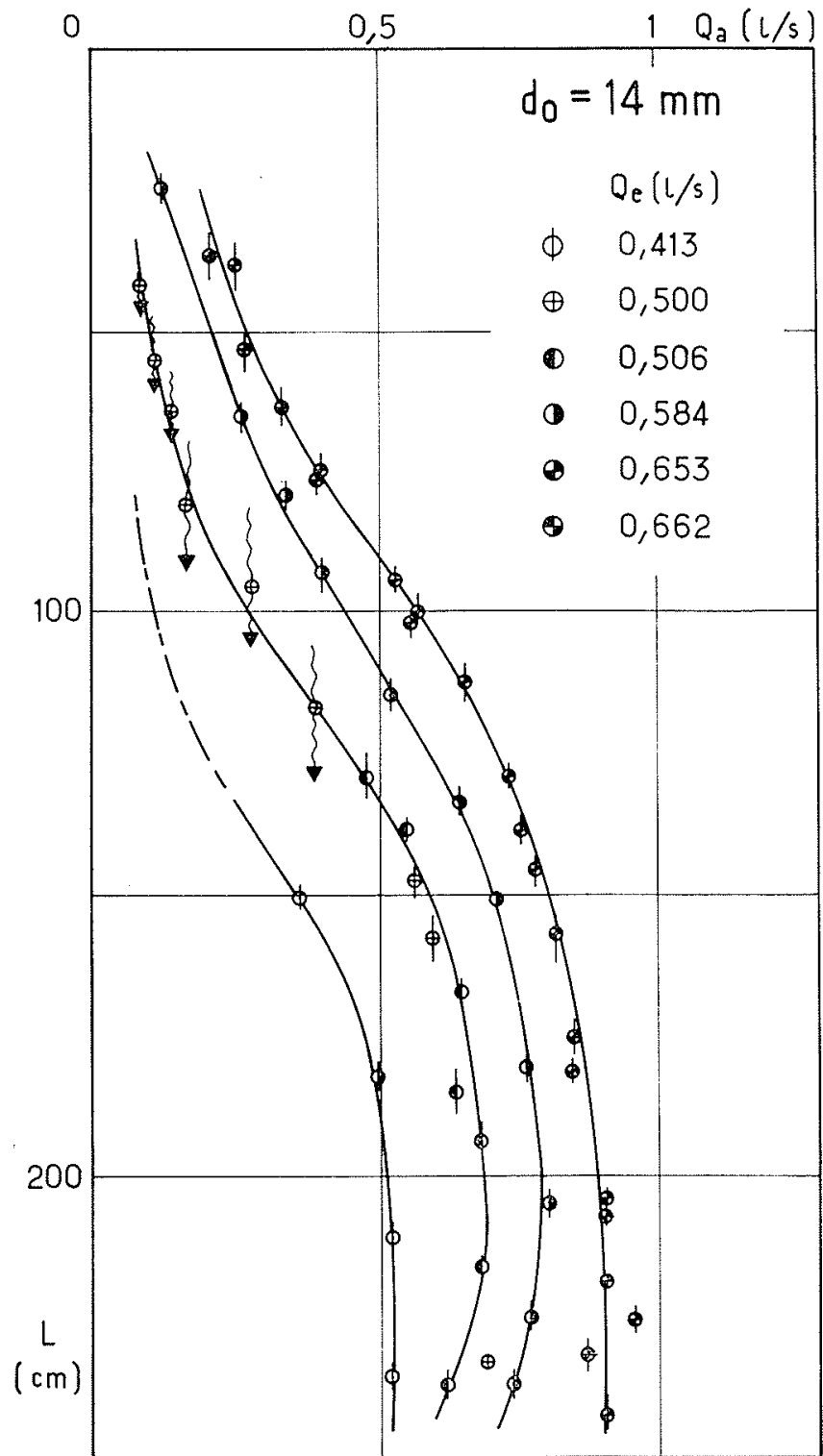

111

10/Relation empirique entre la longueur d'émulsion et la vitesse moyenne de glissement.

Empirical relationship between emulsion lenght and average slip velocity.

11/Transport-Débit d'air. Entrainment-Air flow.

12/Evolution du débit d'air en fonction du débit d'eau pour une longueur de chute libre constante du jet.

Air discharge variation with water discharge, for constant jet free fall height.

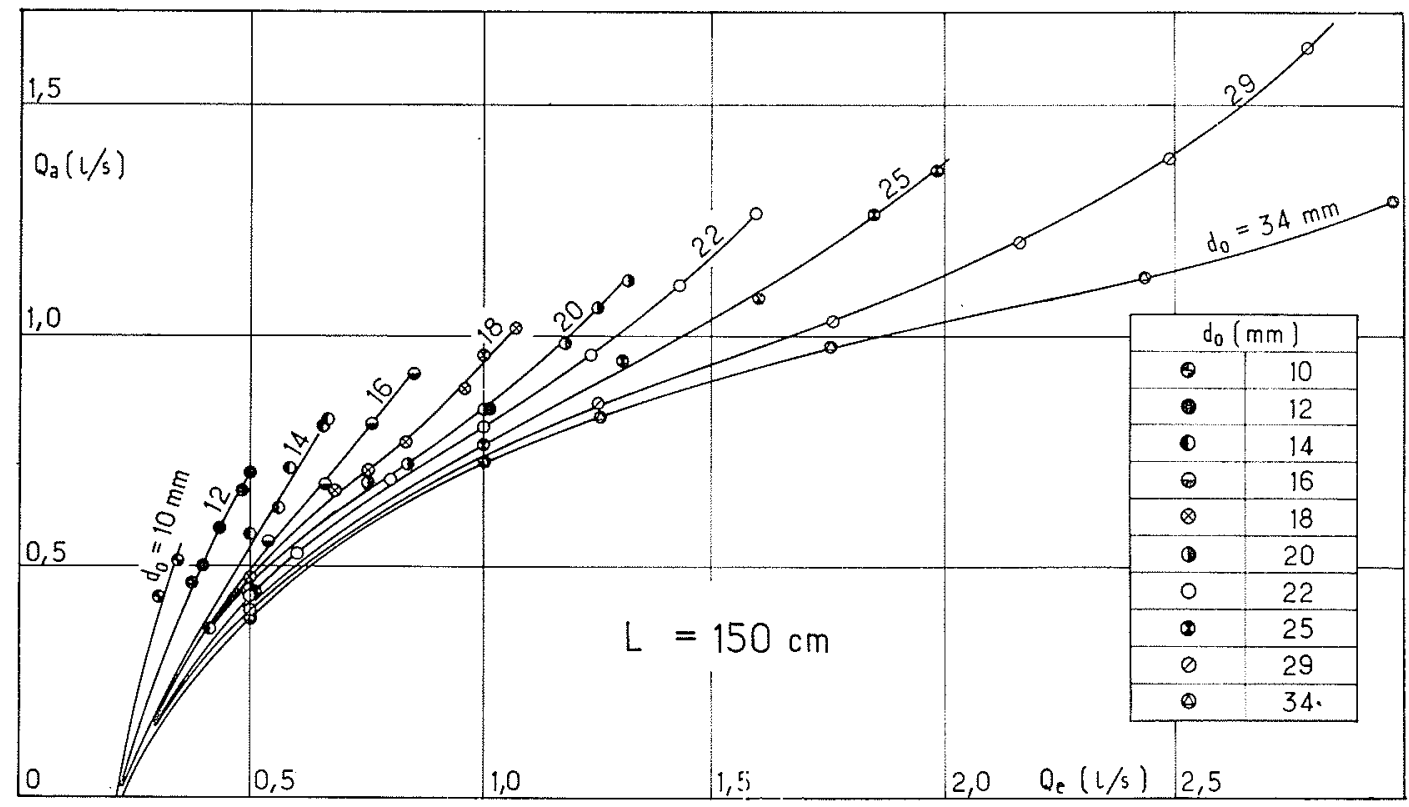




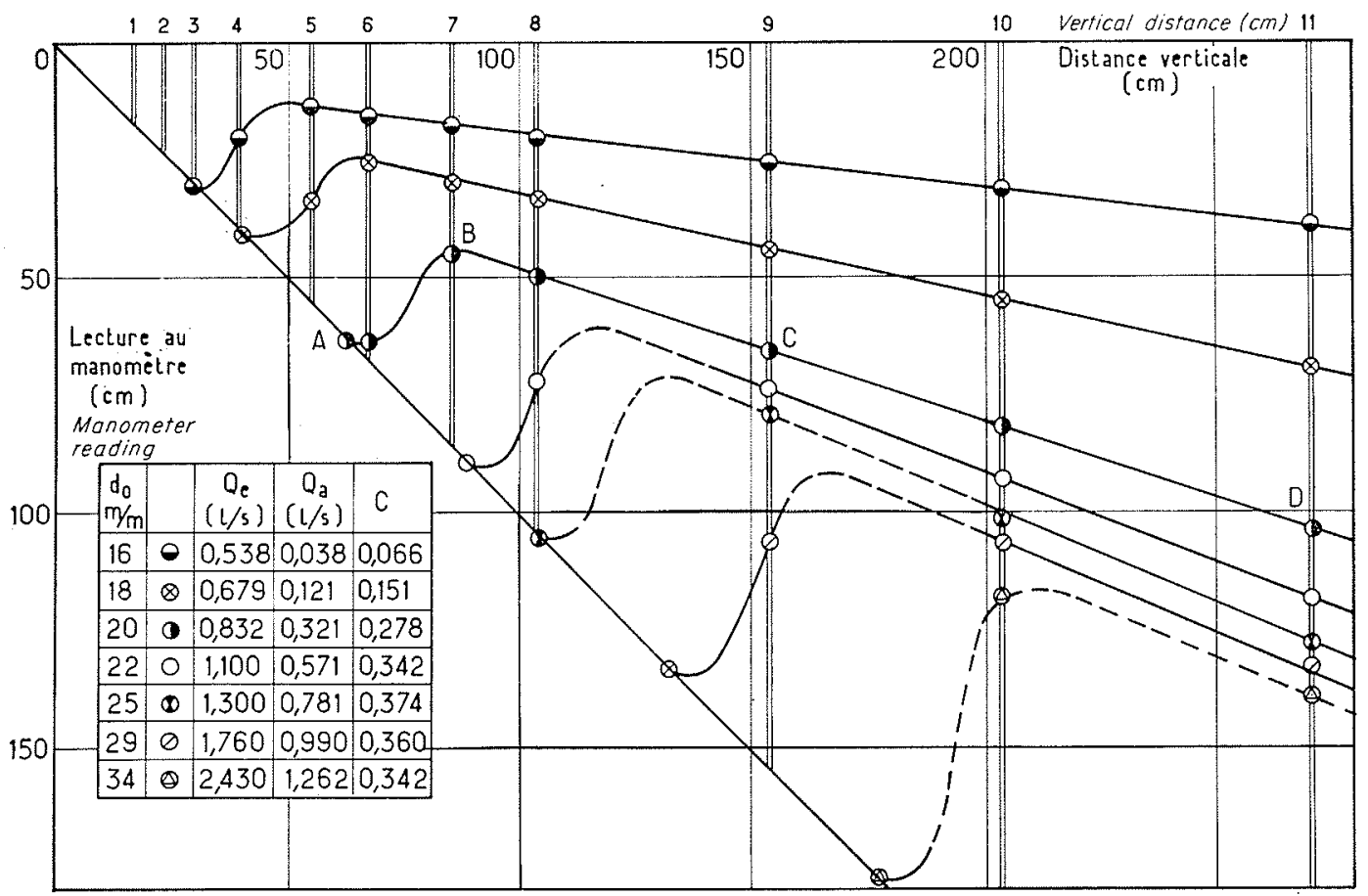

13/ Transport-Pression statique. Entrainment-Static pressure.

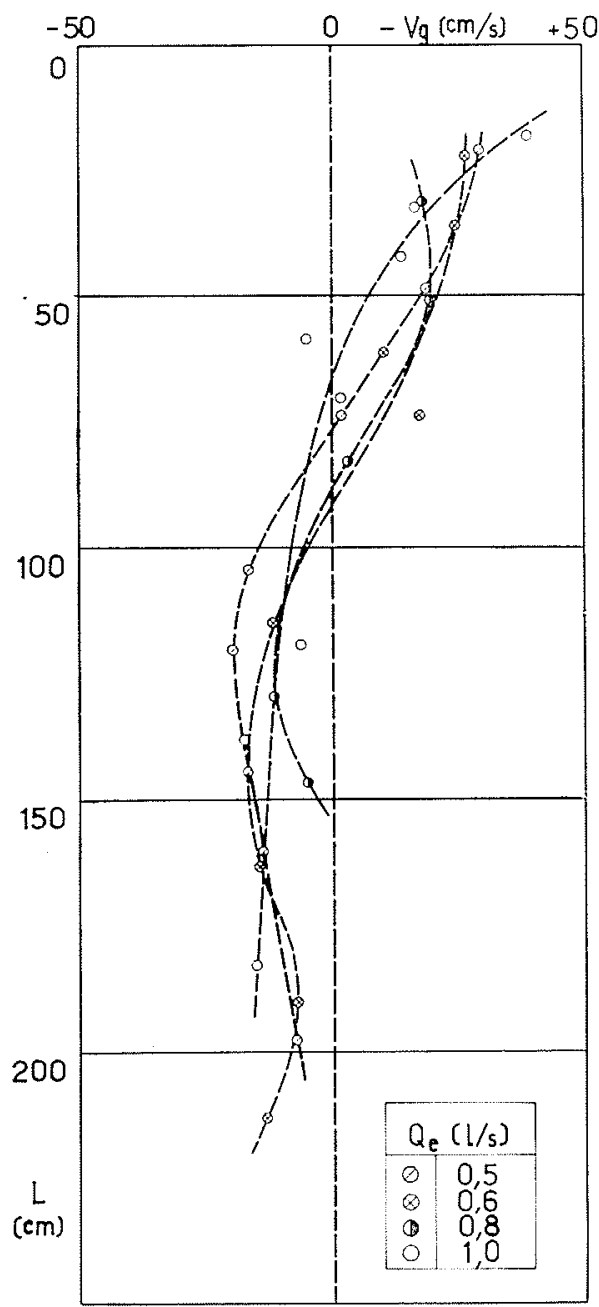

14/ Vitesse moyenne de glissement. Average slip velocity. de montrer que, avec une bonne approximation, on pouvait admettre :

$$
i=c_{m}+\frac{\lambda}{\mathrm{D}} \frac{\mathrm{Q}_{e}{ }^{2}}{2 \mathrm{gS}^{2}\left(1-\mathrm{c}_{m}\right)^{2}}
$$

$\lambda$ étant le coefficient de perte de charge pour l'eau seule $\left(\lambda=0,030\right.$ pour la conduite utilisée), $c_{m}$ étant la concentration moyenne spatiale dans la région du régime établi. On retrouve ainsi une loi de perte de charge dans l'émulsion proche de celle proposée par Mondin [2].

\section{B - Vitesse moyenne de glissement.}

Au cours des essais, nous avons déterminé, en fonction des données, non seulement le débit d'air et la répartition des pressions, mais également la concentration moyenne spatiale. On peut définir une vitesse moyenne de glissement à travers l'émulsion :

$$
\mathrm{V}_{g}=\frac{\mathrm{Q}_{a}}{\mathrm{~S} c_{s}}-\frac{\mathrm{Q}_{e}}{\mathrm{~S}\left(1-c_{s}\right)}
$$

Lorsque le débit d'air est nul, on retrouve la définition de $\mathrm{V}_{g}$ utilisée dans le cas du dégazage.

La figure 14 offre un exemple de variation de $V_{\mathrm{g}}$ en fonction de la longueur de chute $\mathrm{L}$, pour $d_{0}=22 \mathrm{~mm}$ et différents débits. On constate immédiatement que la vitesse de glissement change de signe lorsque $\mathrm{L}$ dépasse une certaine valeur, comprise entre 60 et $90 \mathrm{~cm}$ selon les cas. Sur la foi de ces résultats expérimentaux, il semblerait que, si L est inférieure à cette valeur critique, l'eau s'écoule plus vite que l'air. Par contre, si L est supérieure, la vitesse moyenne de l'air à travers l'émulsion serait plus grande que celle de l'eau.

Ce résultat paraît surprenant et même illogique, étant donné que l'air a toujours tendance à remonter par rapport à l'eau. Aussi, la première fois que 
15 a/ Bouchon ascendant. Rising core.

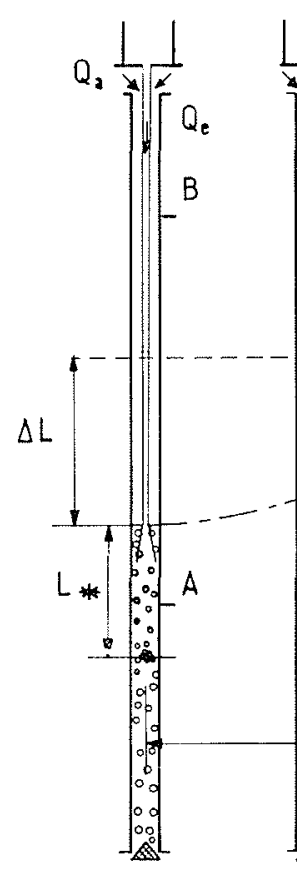

S. 1

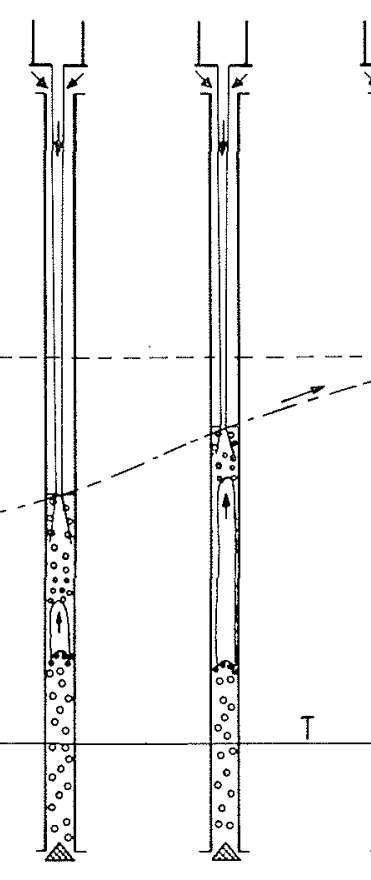

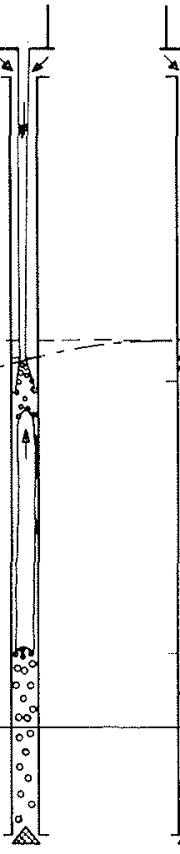

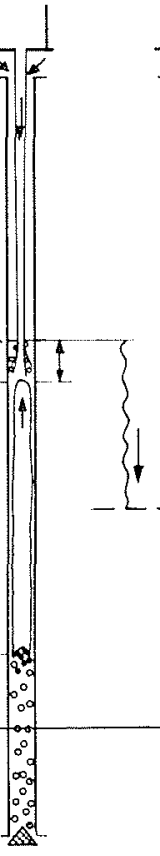

5

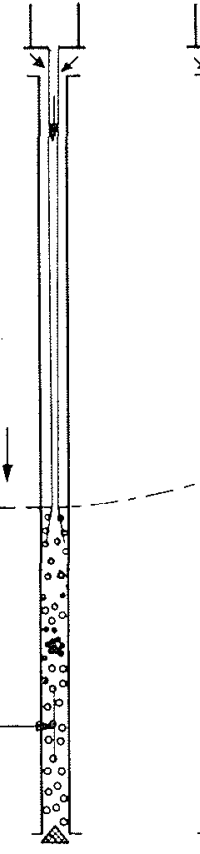

6

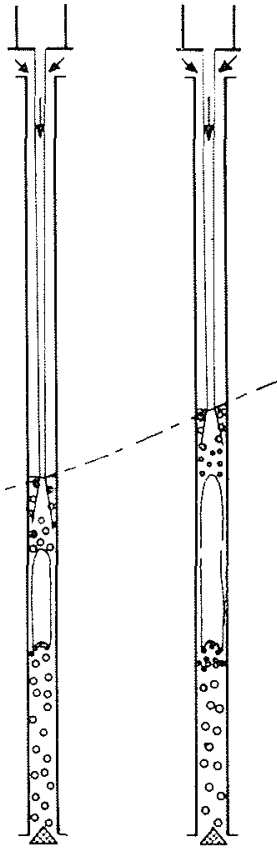

8 nous avons obtenu ce résultat, avons-nous pensé à une erreur expérimentale systématique, portant sur la mesure de la concentration spatiale $c_{s}$ : en effet, cette moyenne porte sur l'ensemble de la colonne en émulsion, y compris la zone de recirculation, et risque d'être différente de la concentration moyenne $c_{m}$ en une section où le régime d'écoulement est dynamiquement établi. Nous verrons plus loin (chap. V) que les résultats d'essais sont parfaitement valables et qu'il faut chercher ailleurs la raison de cette anomalie.

\section{C - Equations générales.}

La simplicité de forme des courbes de pression (fig. 13) suggère un calcul élémentaire du même genre que celui effectué pour le dégazage, dans l'hypothèse de répartitions de vitesse uniformes dans chaque section. La perte de charge $\Delta H$ (fig. $1 b$ ) s'écrit :

$$
\Delta \mathrm{H}=\left(\mathrm{H}^{\prime}-\mathrm{L}\right) c_{s}-\Delta \mathrm{H}_{f}+\Delta \mathrm{H}_{e}
$$

$\Delta \mathrm{H}_{f}$ étant la perte de charge par frottement à travers l'émulsion, $\Delta \mathrm{H}_{e}$ la récupération de pression étoilée due à l'élargissement brusque. De plus :

$$
\left.\mathrm{H}=\mathrm{H}_{1}+\mathrm{L}+\Delta \mathrm{H}+\mathrm{H}_{\mathrm{S}} \text { (d'après la fig. } 1 b\right)
$$

Si l'on fixe $d_{0}$ et $Q_{e}$, la hauteur $\mathrm{H}$ étant donnée, les équations générales sont, comme dans le cas du dégazage, insuffisantes pour décrire le comportement de l'émulsion. En effet, si l'on s'impose la pression $H_{S}$ sur la vanne, la relation (9) s'écrit :

$$
\mathrm{H}-\mathrm{H}_{1}-\mathrm{H}_{\mathrm{S}}=\mathrm{L}+\Delta \mathrm{H}
$$

Les termes du premier membre étant connus, on obtient une relation entre $\mathrm{L}$ et $\Delta \mathrm{H}$; mais ni le débit d'air $Q_{a}$, ni la concentration $c_{s}$ ne peuvent s'en déduire.
Il faut donc, comme pour le dégazage, rechercher d'autres relations éventuelles entre les variables, si l'on veut espérer décrire les phénomènes mis en jeu dans l'émulsion.

\section{D - Recherche de nouvelles relations.}

Les équations générales ne tiennent pas compte de deux faits pourtant essentiels : l'entraînement d'air par le jet d'eau au voisinage de la zone d'impact, et l'écoulement de l'émulsion elle-même.

Si l'on connaissait les lois de l'entraînement d'air par le jet, on pourrait en déduire une relation entre $\mathrm{Q}_{a}, \mathrm{Q}_{e}, d_{0}$ et la longueur d'impact $\mathrm{L}$, la section $\mathrm{S}$ étant par ailleurs connue. Les essais actuels permettent de donner une relation empirique entre ces variables. Pour des valeurs de $L$ assez grandes, $d_{0}$ n'intervient pas, et cette relation est convenablement représentée par la formule :

$$
\mathrm{Q}_{a}{ }^{2}=\mathrm{A}\left(\mathrm{Q}_{e}-\mathrm{Q}_{e}^{\prime}\right)\left(\mathrm{H}_{1}+\mathrm{L}\right) \mathrm{D} \sqrt{g} \mathrm{D}
$$

$\mathrm{D}, \mathrm{H}_{1}$ et $\mathrm{L}$ sont exprimés en $\mathrm{dm}, g$ en $\mathrm{dm} / \mathrm{s} . \mathrm{s}, \mathrm{Q}_{0}$ et $Q_{a}$ en $1 / \mathrm{s}, Q_{e}^{\prime}=0,22 \mathrm{l} / \mathrm{s}, A=0,0125$.

Il nous reste, comme pour le dégazage, à tenter de dégager des lois éventuelles de l'écoulement dans l'émulsion air-eau. Nous allons entreprendre cette étude, après avoir examiné quelques aspects du transport d'air instable.

\section{IV. - Transport d'air instable}

\section{A - Bouchon d'air ascendant.}

Nous avons vu que ce régime pouvait être observé pour des débils d'eau de 0,2 à $0,4 \mathrm{l} / \mathrm{s}$.

Les huit schémas de la figure 15 a donnent une idée de l'évolution du bouchon en fonction $d u$ 


\section{R. CURTET et K. DJONIN}

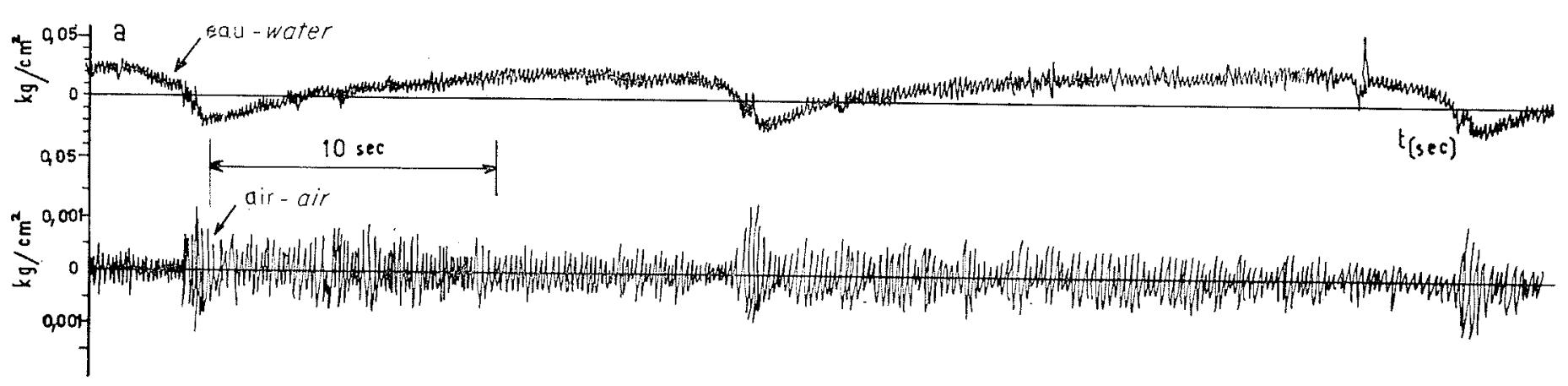

b

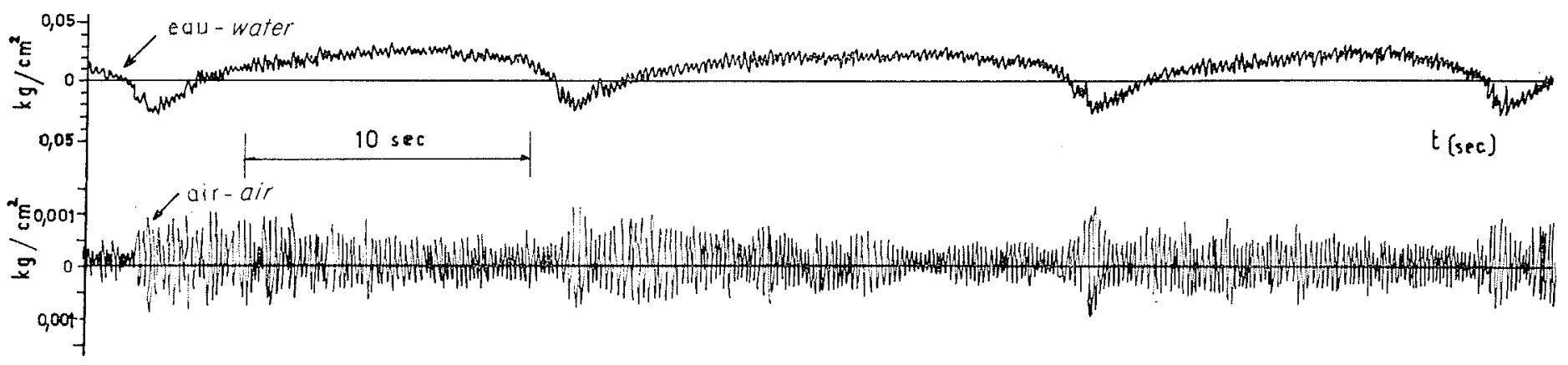

15 b/ Bouchon ascendant. Fluctuations de pression / Rising core. Pressure fluctuation..
a) $d_{0}=10 \mathrm{~mm}$
$Q_{e}=0,350 \mathrm{l} / \mathrm{s}$
$\Delta \mathrm{L}=78 \mathrm{~cm}$
$\mathrm{T}=25,2 \mathrm{~s}$
b) $d_{0}=16 \mathrm{~mm}$
$Q_{e}=0,300 \mathrm{I} / \mathrm{s}$
$\Delta \mathrm{L}=90 \mathrm{~cm} \quad \mathrm{I}=15,7 \mathrm{~s}$

temps; ils sont extraits de photos prises au $1 / 1000^{\circ}$ de seconde. On distingue les phases successives suivantes :

- S-1 : formation du noyau initial par coalescence;

- S-2, 3 et 4 : le bouchon se développe, sa base demeurant localisée à l'endroit où est apparu le noyau; le sommet de la poche monte et entraîne avec lui une partie de l'émulsion, de sorte que la surface d'impact du jet remonte elle aussi;

- S-5 : le sommet du bouchon atteint une altitude maximale et se trouve alors à une distance de la section d'impact du jet d'environ $25 \mathrm{~cm}$, c'est-à-dire de la longueur de recirculation : un paquet d'émulsion demeure ainsi en suspens pendant quelques dixièmes de seconde et retombe ensuite sur la base de la poche qui, de ce fait, disparaît;

- S-6 : un nouveau noyau se reforme et le cycle recommence.

La surface libre évolue entre deux niveaux distants de $\Delta \mathrm{L}$; L. est la distance du point de coalescence au niveau le plus bas de la surface libre.

Nous avons ménagé à la paroi de la conduite deux prises de pression statique, que nous avons raccordées à des capteurs à membrane réunis à un enregistreur. L'une des prises était placée au point A dans l'émulsion (fig. 15 a), l'autre en $B$ dans l'air, au-dessus de l'émulsion.

La figure $15 b$ représente deux exemples d'enregistrement de la pression statique en fonction du temps : l'allure générale des courbes est la même dans les deux cas, bien qu'elles correspondent à des débits d'eau et d'air différents. On retrouve d'abord le caractère à peu près périodique du phénomène, et les discontinuités provoquées par la destruction de la poche (fig. $15 a$, S-5 et 6 ). Au cours de la formation et de l'extension du bouchon, la pression statique augmente en A puisque la surface d'impac: du jet remonte; puis, lorsque le sommet de la bulle atteint la prise, la pression devient constante et sensiblement égale à la pression qui règne à l'intérieur du bouchon. Enfin la disparition de la bulle provoque une brusque décroissance de la pression.

Dans l'air, on retrouve ces discontinuités, qui s'accompagnent d'ailleurs de variations brusques du débit d'air, décelées au compteur. On note que les fluctuations de pression sont élevées, même dans l'air entourant le jet.

Le développement du bouchon s'accompagne de fortes fluctuations turbulentes de l'écoulement diphasé et la durée de vie d'un bouchon peut varier notablement pour des conditions données. Il est cependant possible de considérer une valeur moyenne de cette duxée pendant un temps assez long et de définir ainsi une période moyenne $T$ du cycle schématisé sur la figure $15 a$. Nous avons rassemblé sur la figure 16 quelques résultats d'essais, montrant la variation de la période $\mathrm{T}$ en fonction de l'amplitude $\Delta \mathrm{L}$ et du débit d'eau $\mathrm{Q}_{\epsilon}$, pour quelques diaphragmes.

\section{B - Bouchon d'air descendant.}

Lorsque le débit d'eau est supérieur à $0,5 \mathrm{l} / \mathrm{s}$, on n'observe jamais de bouchon ascendant, ni de poche stationnaire (fig. 3); il est par contre possible d'observer l'évolution d'un bouchon descendant, dont quelques aspects sont représentés sur la figure $17 a$ : 
- S-3 : formation du noyau et coalescence;

- S-4, 5, 6: la bulle se développe et descend simultanément, cependant que la surface d'impact du jet s'élève;

- S-7 : la base du bouchon se trouve à proximité de la vanne-pointeau et la surface libre est à sa cote maximale. La poche d'air sort de la conduite à travers la vanne et le niveau d'impact s'abaisse brusquement pendant le même temps;

- S-8 : le cycle recommence.

La figure $17 b$ offre un exemple d'enregistrement de la pression statique en A et B (fig. 15a) : on retrouve les discontinuités dues à la disparition de la poche, mais les fluctuations de pression sont telles qu'il n'est pas possible d'observer un palier au passage de la bulle.

\section{C - Bouchon d'air stalionnaire.}

Entre 0,4 et $0,5 \mathrm{l} / \mathrm{s}$, il apparaît un régime intermédiaire entre les bouchons ascendant et descendant, le bouchon d'air stationnaire; il se forme au début de la même manière que le bouchon ascendant, mais, à partir d'un certain instant, sa géométrie cesse d'évoluer.

Nous nous proposons de comparer ce type d'écoulement au schéma classique de la bulle s'élevant à l'intérieur d'un tube (fig. $18 \mathrm{a}$ ), dans un liquide supposé parfait, c'est-à-dire sans viscosité.

Rappelons à ce propos les travaux théoriques et expérimentaux de Dumitrescu [3], dont les résultats ont été sensiblement retrouvés par Davies et Taylor [4] qui ont utilisé un autre mode de raisonnement. Ces auteurs trouvent un bon accord entre les calculs et les expériences: près du point $B$ (fig. $18 a$ ) la surface de la bulle se confond sensiblement avec une calotte sphérique et la vitesse de

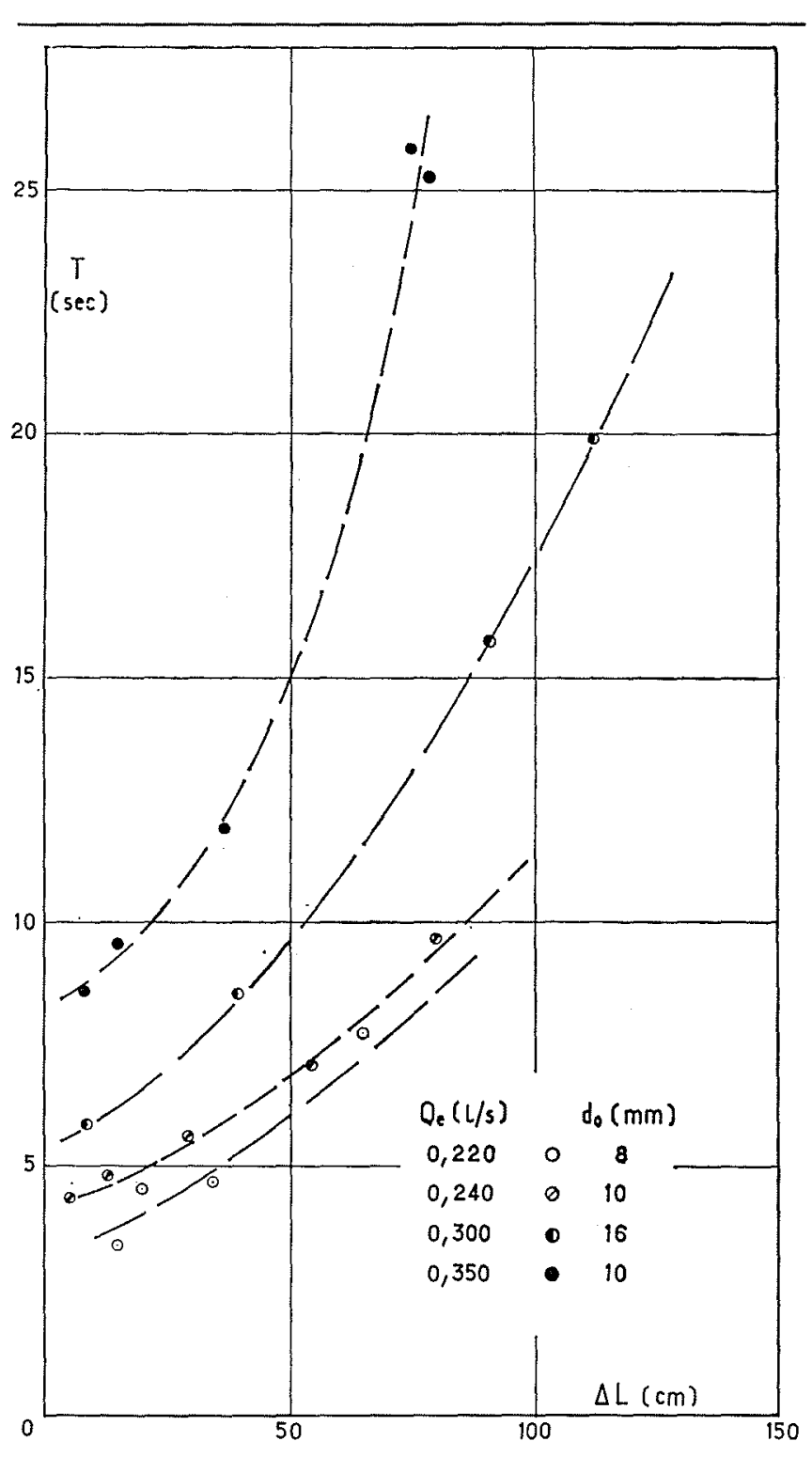

$16 /$

Période du bouchon ascendant

Rising core period.

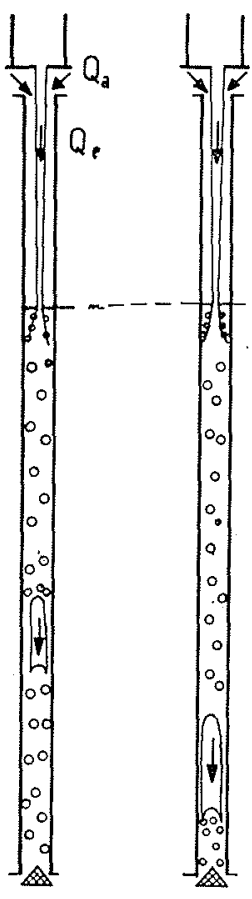

S. 1

2

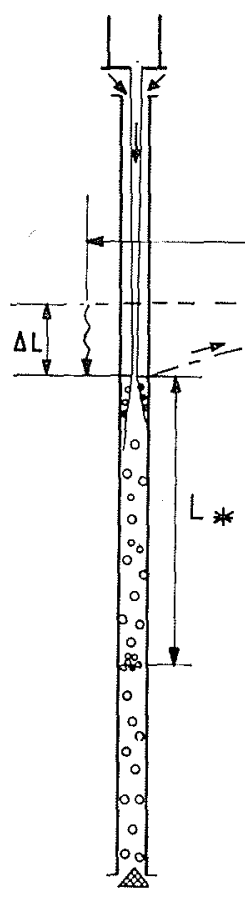

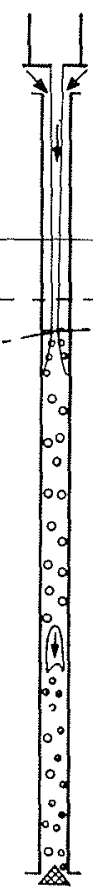

4

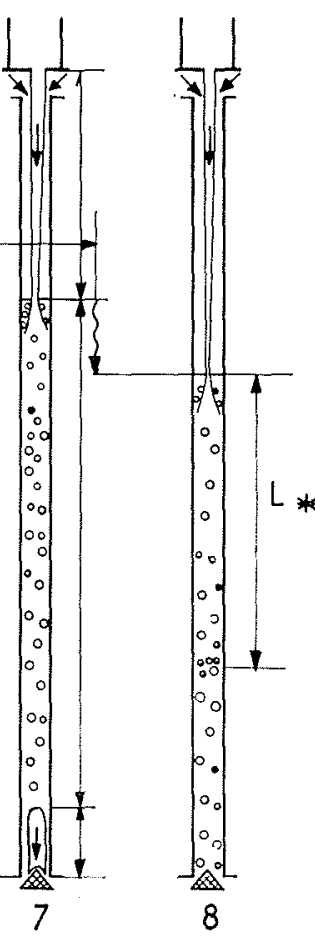

17 a/

Bouchon descendant. Sinking core. 


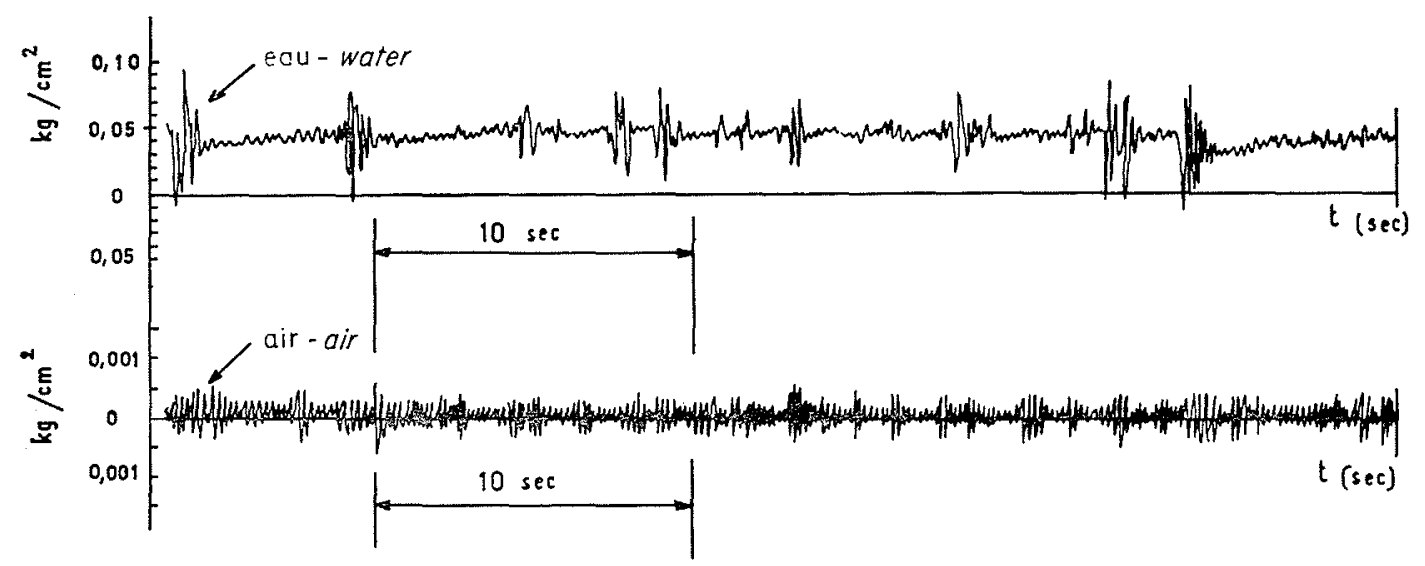

17 b/

Bouchon descendant. Fluctuations de pression.

Sinking core. Pressure fluctuations.

$\mathrm{Q}_{e}=0,480 \mathrm{l} / \mathrm{s} \quad \Delta \mathrm{L}=21 \mathrm{~cm}$ $\mathrm{T}=5,2 \mathrm{~s}$ déplacement $V_{b}$ de la bulle par rapport au fluide est une fonction du rayon $R$ du tube :

$$
\mathrm{V}_{b}=\alpha \sqrt{g} \overline{\mathrm{R}}
$$

Dumitrescu trouve $\alpha=0,496$ par le calcul, et $\alpha=0,49$ par l'expérience. Nous avons reporté sur la figure $18 b$ les résultats des mesures des auteurs cités, ainsi que nos valeurs expérimentales de $V_{b}$ pour le bouchon stationnaire et pour $\mathrm{D}=46,4 \mathrm{~mm}$. En fluide parfait, on devrait avoir $\mathrm{V}_{b}=0,24 \mathrm{~m} / \mathrm{s}$, alors que nous trouvons des valeurs comprises entre 0,23 et $0,35 \mathrm{~m} / \mathrm{s}$.
Nous avons calculé la vitesse relative $V_{b}$ en l'admettant égale à $\left(Q_{e}+Q_{a}\right) / S$, c'est-à-dire à la vitesse moyenne de l'émulsion. Plus généralement, nous avons considéré un bouchon se déplaçant à la vitesse absolue $\mathrm{V}_{s}$ le long de la conduite. Compte tenu de l'équation (11), on peut écrire, comme Zuber et Findlay par exemple [6]:

$$
\mathrm{V}_{s}=\frac{\mathrm{Q}_{e}+\mathrm{Q}_{u t}}{\mathrm{~S}}-\alpha \sqrt{g} \mathrm{R}
$$

Nous avons rassemblé sur la figure $18 c$ un certain nombre de nos résultats d'essais, en portant en abs-
18 a/
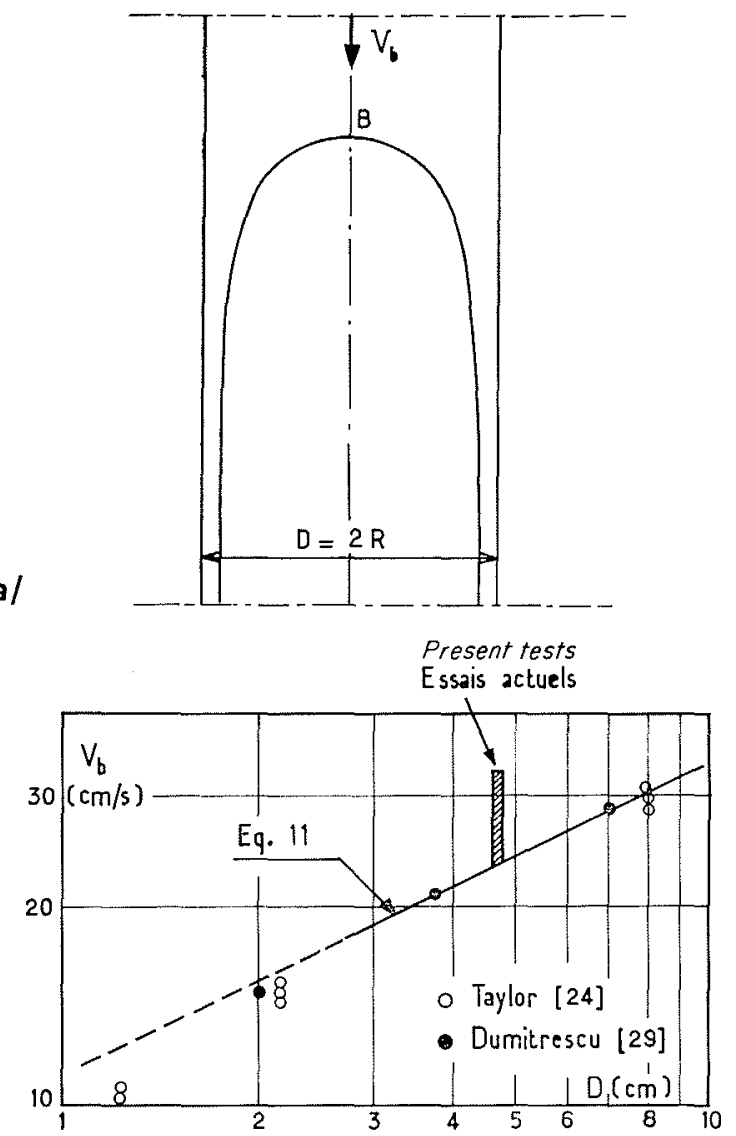

18 b/ Comparaison des résultats d'essais à des études théoriques (bouchon stationnaire).

Comparison between test results and theoretical data (stationary core).

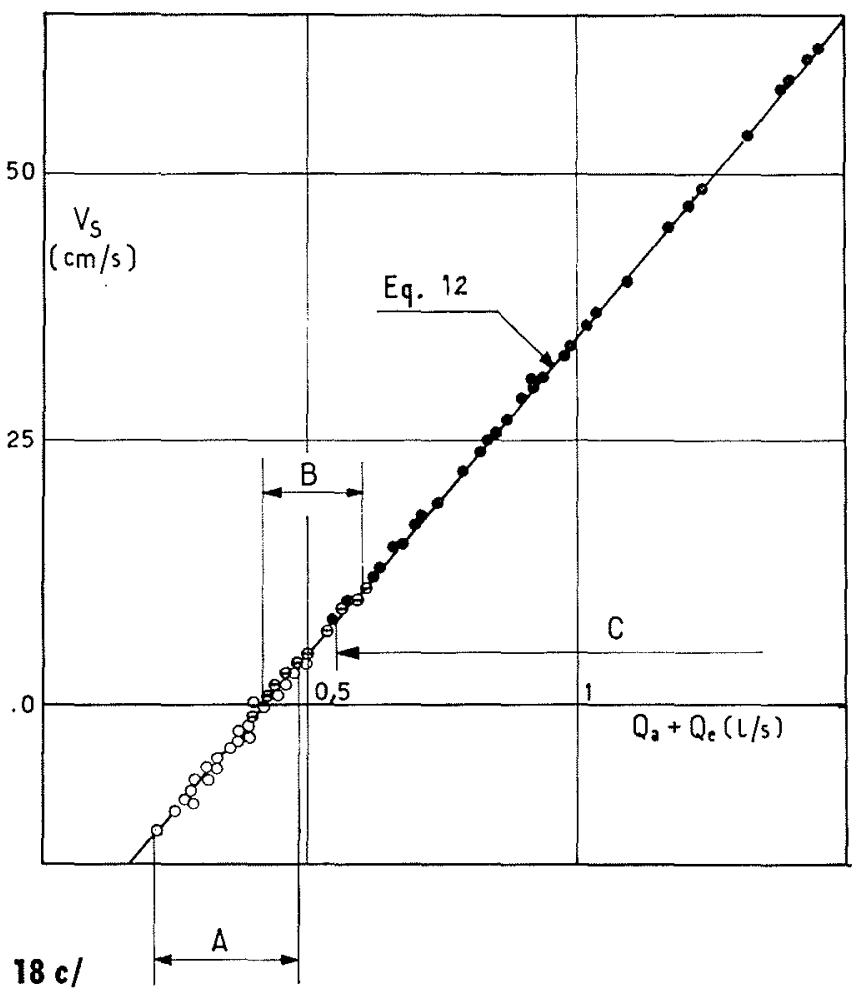

A. Bouchon ascendant. Rising core.

B. Bouchon stationnaire. Stationary core.

C. Bouchon descendant. Sinking core. 
cisses le débit total de l'émulsion $\left(Q_{a}+Q_{c}\right)$ et en ordonnées la vitesse $\mathrm{V}_{s}$ calculée à partir de l'équation (12). On devrait obtenir, pour $V_{s}<0$, un bouchon ascendant, $V_{s}=0$ un bouchon stationnaire, et $\mathrm{V}_{s}>0$, un bouchon descendant. On retrouve effectivement un classement de ce genre, ce qui permet de justifier le rôle prépondérant joué par le débit de l'émulsion sur le déplacement des poches instables et de confirmer ainsi une remarque que nous avions déjà formulée à propos de la figure 3 .

\section{V. - Analyse des profils de concentration}

Nous venons de voir que, pour le dégazage comme pour le transport, il manque une équation liée au comportement de l'émulsion. S’il était possible de trouver une relation de ce genre, nous ferions un grand pas vers la résolution du problème posé initialement.

\section{A - Définitions ef notations.}

La figure 19 résume les principales notations qui vont nous être utiles. A l'aide de la méthode aux rayons gamma, nous mesurons une concentration moyenne $\bar{c}$ le long de la corde $A B$; cette corde, de longueur $l$, est parallèle à l'axe $y^{\prime} y$ et perpendiculaire à l'axe $x^{\prime} x$, le long duquel est déplacé le cha- riot de mesure. Soit $x$ l'abscisse et $\mathrm{Y}$ l'ordonnée de $\mathrm{B}: x$ varie de $-\mathrm{R}$ à $+\mathrm{R}, \mathrm{Y}$ de 0 à $\mathrm{R}$; $c$ étant la concentration en un point d'abscisse $x$ et d'ordonnée $y$, la concentration marginale $\bar{c}$ est définie par :

$$
\bar{c}=\int_{0}^{\mathrm{Y}} c \frac{d y}{\mathrm{Y}}
$$

La distribution de concentration $c$ en fonction du rayon $r$ présente dans nos essais l'allure donnée sur la figure $19, c$ étant nulle à la paroi de la conduite et maximale $\left(c=c_{c}\right)$ au centre. Il en est de même pour la concentration marginale dont la valeur sur l'axe est $\bar{c}_{c}$.

\section{B - Courbes expérimentales et représentation analytique.}

Nous donnons sur la figure 20 deux séries de profils de concentration, obtenus avec le diaphragme de $22 \mathrm{~mm}$. Chaque profil correspond aux débits d'eau et d'air indiqués sur les tableaux portés sur la figure 20, ainsi que la moyenne $c_{m}$ des concentrations dans la section. Les concentrations $c_{m}$ et $\bar{c}$ sont liées par la relation :

$$
\frac{\pi \mathrm{R}^{2}}{4} c_{m}=\int_{0}^{\mathrm{R}} \bar{c} \mathrm{Y} d x
$$

La figure 21 montre l'évolution de $c_{m}$ en fonction de la vitesse moyenne de l'émulsion, pour différents

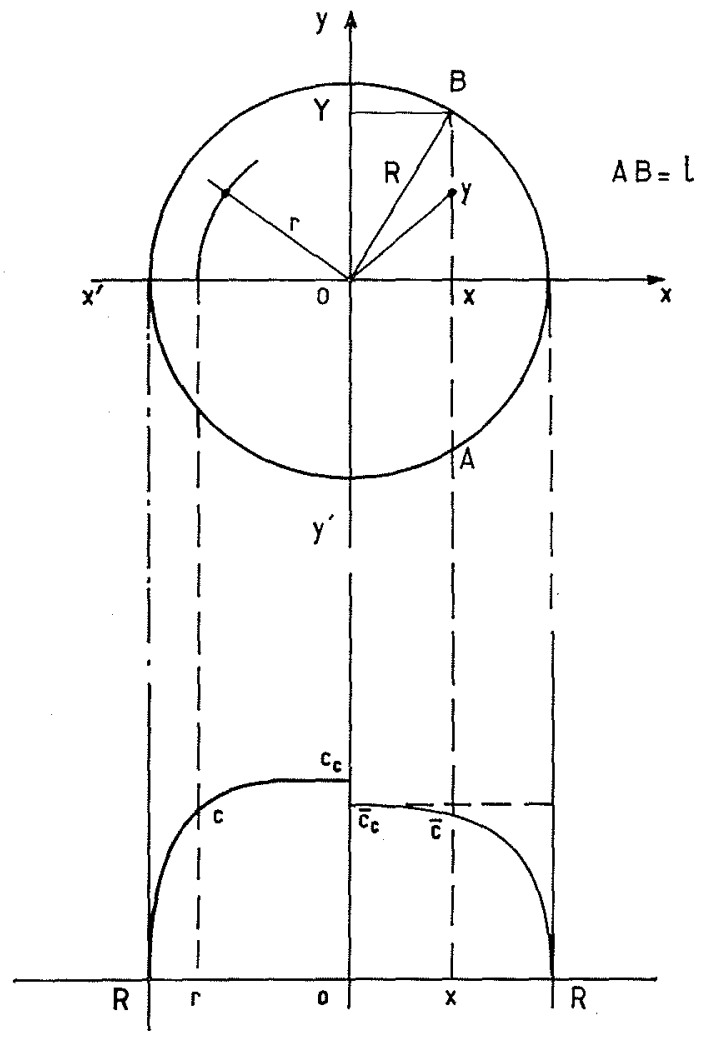

19/ Mesures de concentration. Notations. Concentration measurement notation.

20/ Courbes expérimentales de concentration. Experimental concentration curves.

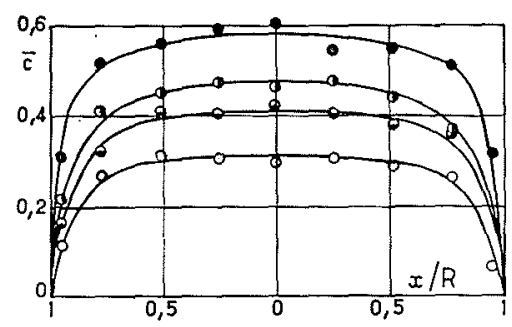

C $\quad d_{a}=22 \mathrm{~mm} \quad O_{e}=1,130 \mathrm{l} / \mathrm{s}$

\begin{tabular}{|r|r|c|c|}
\hline & $L(\mathrm{~cm})$ & $0_{\mathbf{2}}(\mathrm{L} / \mathrm{s})$ & $c_{\mathrm{m}}$ \\
\hline 0 & 50 & 0,447 & 0,284 \\
\hline 0 & 100 & 0,700 & 0,378 \\
\hline 0 & 150 & 0,877 & 0,434 \\
\hline 0 & 200 & 1,000 & 0,546 \\
\hline
\end{tabular}

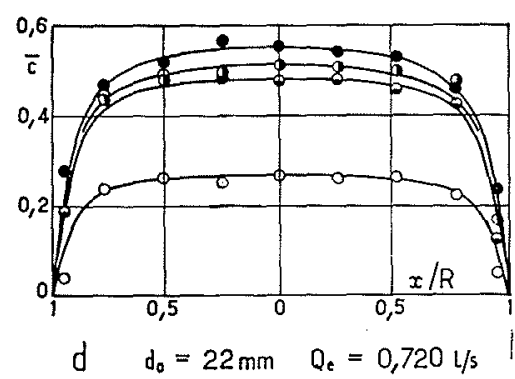

\begin{tabular}{|c|c|c|c|}
\hline & $L(c \mathrm{~cm})$ & $Q_{\mathrm{a}}(\mathrm{L} / \mathrm{s})$ & $\mathrm{c}_{\mathrm{m}}$ \\
\hline 0 & 50 & 0,192 & 0,246 \\
\hline 0 & 125 & 0,538 & 0,448 \\
\hline 0 & 150 & 0,583 & 0,470 \\
\hline 0 & 200 & 0,620 & 0,510 \\
\hline
\end{tabular}




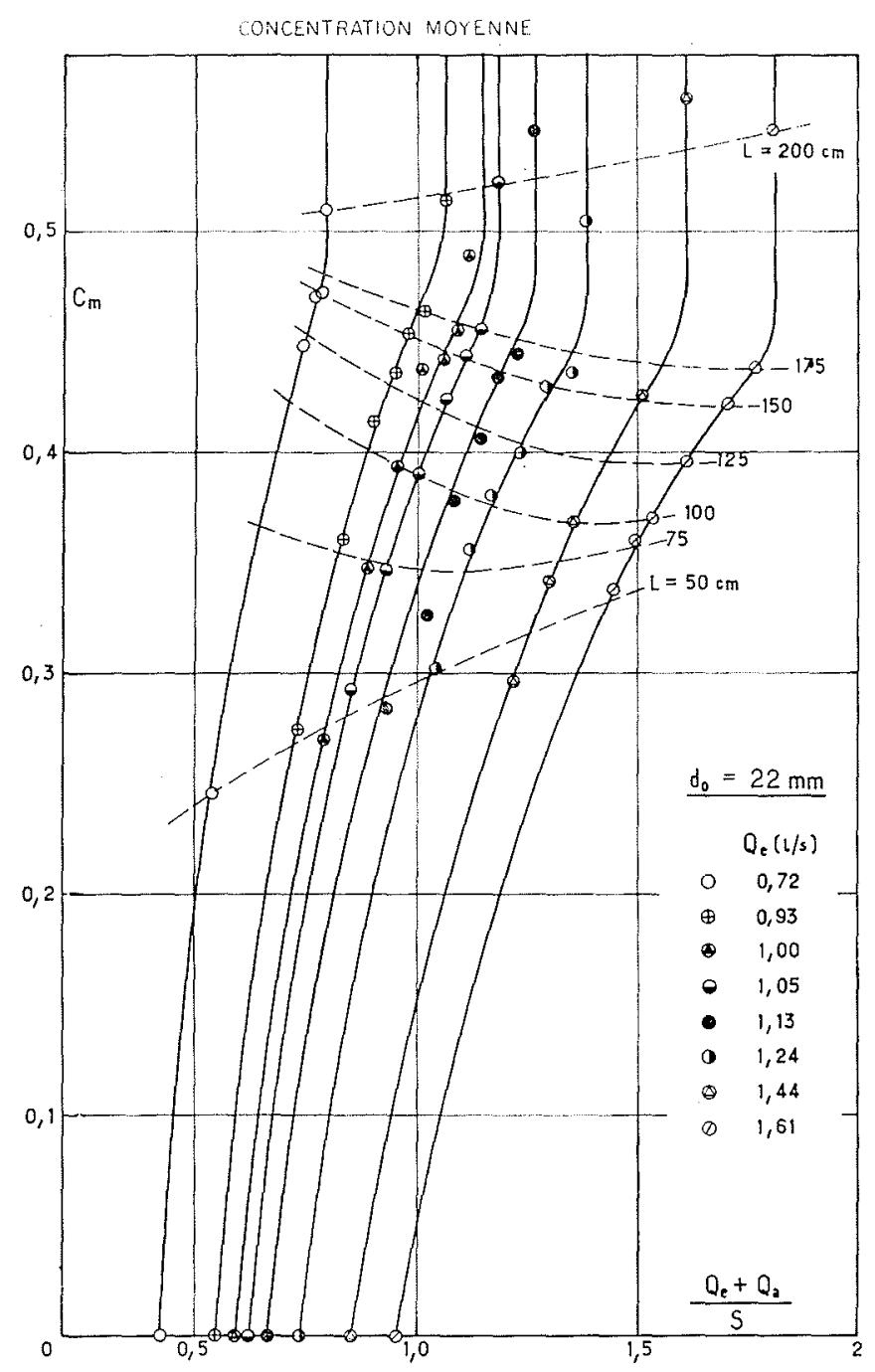

21/

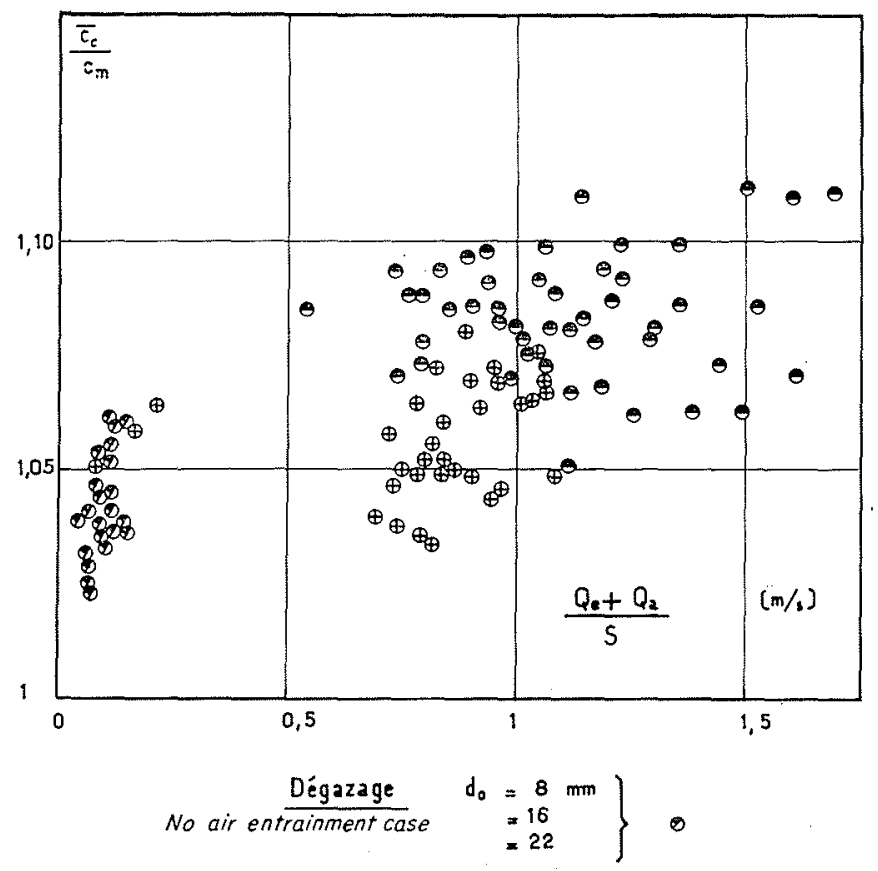

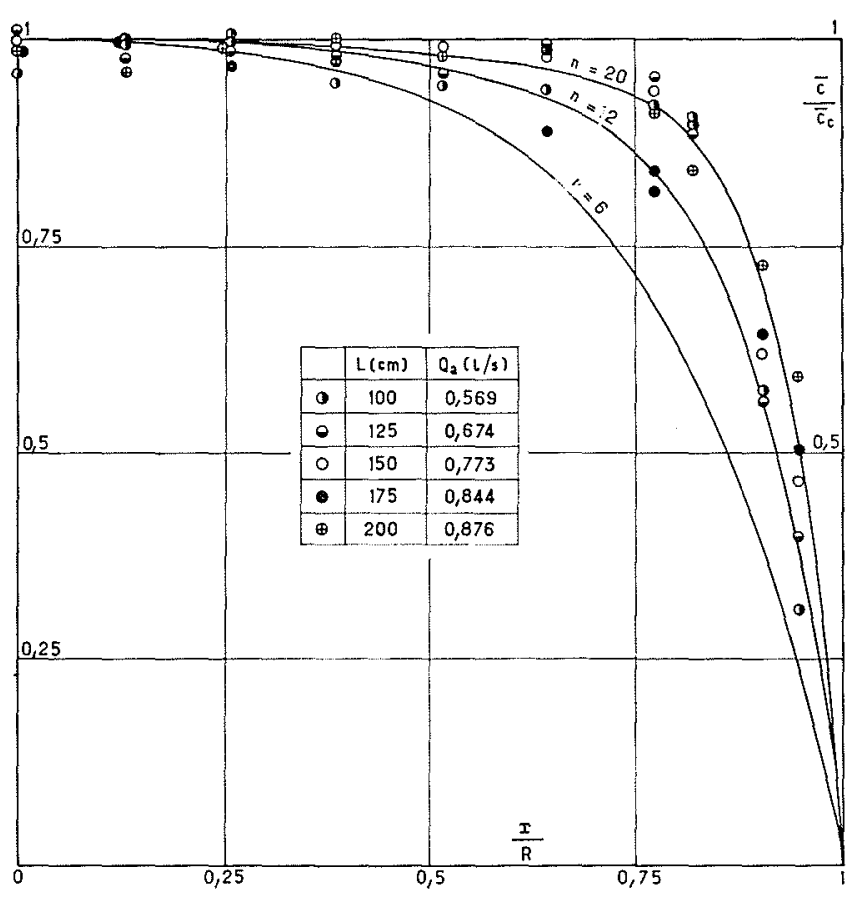

23/

$$
d_{0}=16 \mathrm{~mm} \quad Q_{c}=0,759(\mathrm{~L} / \mathrm{s})
$$

24/

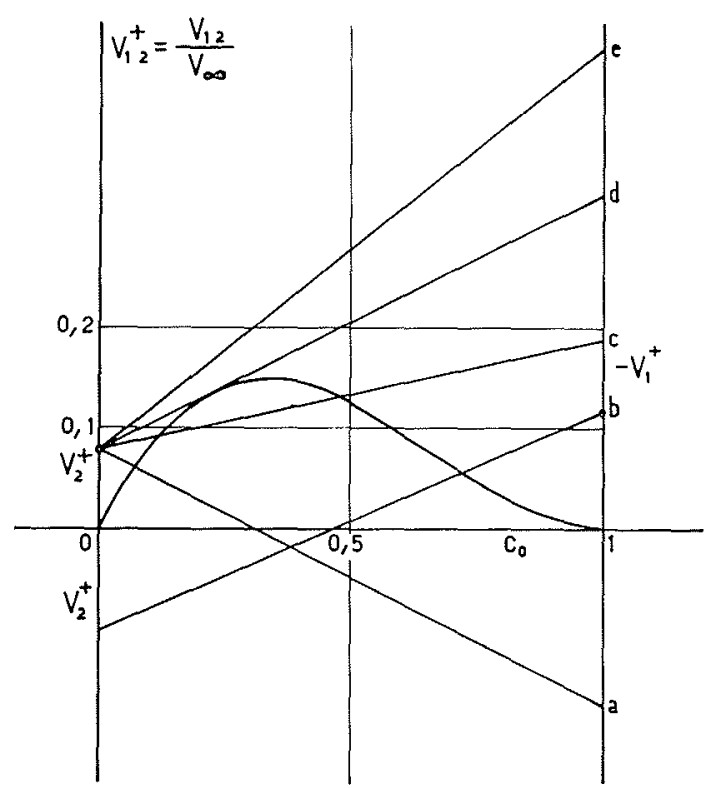

21/ Concentration moyenue. Average concentration.

22/ Evolution du rapport des concentrations $\bar{c}_{v} / c_{m_{2}}$ en fonction de la vitesse moyenne de l'émulsion.

Concentration ratio $\overline{\mathrm{c}}_{\mathrm{e}} / \mathrm{c}_{\mathrm{m}}$ in terms of mean emulsion velocity.

23/ Profils réduits de concentration.

Non-dimensional concentration curves.

24/ Diagramme de Wallis. Wallis diagram. 
débits d'eau; au fur et à mesure que la distance d'impact $L$ croît, le débit d'air transporté et la concentration $c_{m}$ augmentent. Lorsque $\mathrm{L}$ dépasse $190 \mathrm{~cm}$ environ, la section de mesure des concentrations atteint la région de recirculation, le débit d'émulsion demeure constant, mais $c_{m}$ continue à croître.

Sur la figure 22, nous avons porté, en fonction de la vitesse moyenne d'émulsion, le rapport $\bar{c}_{c} / c_{m}$ de la concentration marginale sur l'axe à la concentration moyenne : ce rapport variant relativement peu (entre 1,03 et 1,11 ) nous avons fortement dilaté l'échelle des ordonnées afin de faciliter les lectures. Il en résulte une dispersion des points d'essais, mais le centre de gravité du nuage de points pour le dégazage se situe vers 1,04 (fig. 22) alors que celui correspondant au transport est placé aux alentours de 1,075. Cette tendance est confirmée par l'allure des profils expérimentaux, et montre que, dans le cas du dégazage, on se rapproche très nettement d'une distribution uniforme des concentrations, alors que pour le transport, on s'en éloigne d'autant plus que le débit d'émulsion est plus élevé.

Certains auteurs ont proposé des formules pour représenter les profils de concentration; Zuber et Findlay [6] notamment préconisent la relation :

$$
\frac{c}{c_{c}}=1-\left(\frac{r}{\mathrm{R}}\right)^{n}
$$

Cette formule nous a permis d'exprimer $\bar{c}$ en fonction de $c$, d'après l'équation (13):

$$
\bar{c}=\frac{c_{o}}{\mathrm{Y}} \int_{0}^{\mathrm{Y}}\left[1-\left(\frac{r}{\mathrm{R}}\right)^{n}\right] d \mathrm{y}
$$

et de déterminer la répartition de $\bar{c}$ en fonction de $x / \mathrm{R}$ pour différentes valeurs de $n$. La comparaison des courbes ainsi calculées aux profils expérimentaux, comme le montre par exemple la figure 23 , montre tout d'abord que la représentation de Zuber (15) est relativement proche de la réalité; on constate par ailleurs que les valeurs du coefficient $n$ sont élevées, entre 8 et 20 pour le transport, de 15 à 30 pour le dégazage.

Ces données étant maintenant connues, nous allons examiner deux modèles classiques proposés pour l'étude des écoulements à deux phases en régime établi, l'un dû̀ à Wallis [5] l'autre à Zuber et Findlay [6].

\section{C - Schéma de Wallis [5]}

Wallis étudie les propriétés globales d'un système particulaire, formé par la dispersion d'une phase discontinue 2 dans une phase continue 1, la dimension des particules étant faible devant le diamètre de la conduite. Il considère un écoulement où la répartition des vitesses d'eau et d'air et des concentrations est uniforme en chaque section. La vitesse de glissement $V_{g}$ est :

$$
\mathrm{V}_{g}=\frac{\mathrm{Q}_{2}}{\mathrm{~S} c_{0}}-\frac{\mathrm{Q}_{1}}{\mathrm{~S}\left(1-c_{0}\right)}
$$

$S$ étant la section, $Q_{1}$ et $Q_{2}$ les débits des phases continue et dispersée, $c_{0}$ la concentration supposée uniforme de 2 dans 1. Après multiplication de (17) par $c_{0}\left(1-c_{0}\right)$, Wallis obtient une vitesse caractéristique $\mathrm{V}_{12}$ telle que :

$$
\mathrm{V}_{12}=\mathrm{V}_{g} c_{0}\left(1-c_{0}\right)=\mathrm{V}_{2}\left(1-c_{0}\right)-\mathrm{V}_{1} c_{0}
$$

$\mathrm{V}_{1}=\mathrm{Q}_{1} / \mathrm{S}$ et $\mathrm{V}_{2}=\mathrm{Q}_{2} / \mathrm{S}$ sont les vitesses superficielles ou apparentes des phases 1 et 2 .

L'auteur admet que le diamètre des grains ou des bulles est uniforme; soit $V_{\infty}$ la vitesse d'une particule isolée dans le milieu 1 supposé indéfini. L'auteur propose une relation entre la vitesse de glissement et la concentration $c_{0}$, de la forme :

$$
\mathrm{V}_{g}=\mathrm{V}_{\infty}\left(1-c_{0}\right)^{k}
$$

de sorte que, d'après (18):

$$
V_{12}=V_{\infty} c_{0}\left(1-c_{0}\right)^{k+1}
$$

Pour des bulles de diamètre moyen compris entre $1 \mathrm{~mm}$ et $2 \mathrm{~cm}, V_{\infty}=0,26 \mathrm{~m} / \mathrm{s}$. Par ailleurs, le coefficient $j$, selon les auteurs, varie entre 0 et 2 .

\section{a) Diagramme de Wallis.}

Le diagramme de Wallis, présenté sous forme adimensionnelle (fig. 24), comporte en abscisses la concentration $c_{0}$, et en ordonnées la vitesse caractéristique $\mathrm{V}_{12}{ }^{+}=\mathrm{V}_{12} / \mathrm{V}_{\infty}$. Appelons :

$$
\mathrm{V}_{1}^{+}=\mathrm{Q}_{1} / \mathrm{SV}_{\infty} \text { et } \mathrm{V}_{2}{ }^{+}=\mathrm{Q}_{2} / \mathrm{SV}_{\infty}
$$

l'équation (21) déduite de (18) :

$$
\mathrm{V}_{1}{ }^{+}=\mathrm{V}_{2}{ }^{+}\left(1-c_{0}\right)-\mathrm{V}_{1}{ }^{+} c_{0}
$$

se traduit par une droite passant par les points 0 , $\mathrm{V}_{2}{ }^{+}$et $1,-\mathrm{V}_{1}{ }^{+}$. L'expression $(20)$, pour $k=1$, conduit à la courbe portée sur la figure 24 .

L'intersection de la droite et de la courbe donne une solution du problème. Considérons par exemple une émulsion d'air dans l'eau, pour laquelle la vitesse réduite $\mathrm{V}_{12}{ }^{+}$est nécessairement dirigée ver's le haut; plusieurs configurations sont possibles, selon la figure 24 :

\section{$1^{\circ}$ Ecoulement concourant :}

- l'air et l'eau montent : droite $a, \mathrm{~V}_{2}{ }^{+}>0, \mathrm{~V}_{1}{ }^{+}>0$ donc $-\mathrm{V}_{1}{ }^{+}<0$;

- l'air et l'eau descendent, ce qui est notre cas : droite $b, \mathrm{~V}_{2}{ }^{+}<0, \mathrm{~V}_{1}{ }^{+}<0$ donc $-\mathrm{V}_{1}{ }^{+}>0$.

Dans les deux cas, la droite coupe la courbe en un seul point d'intersection : il $\mathrm{y}$ a une solution et une seule.

\section{$2^{\circ}$ Ecoulement ḋ contre-courant :}

Il n'est pas possible que l'air descende et que l'eau monte simultanément. Il reste une possibilité : l'air s'élève et l'eau descend, d'où $\mathrm{V}_{2}{ }^{+}>0$, et $\mathrm{V}_{1}{ }^{+}<0$, donc $-\mathrm{V}_{1}{ }^{+}>0$. Selon les valeurs de $\mathrm{V}_{1}{ }^{+}$ et de $\mathrm{V}_{2}{ }^{+}$, on peut obtenir deux solutions (droite $c$ ), une solution limite (droite $d$ ) ou même ne pas avoir de solution (droite $e$ ) : la condition limite $d$ est appelée «flooding » par Wallis et doit correspondre, selon cet auteur, à un changement de structure de l'écoulement, par disparition d'une phase, pulsations, poches d'air (ou « slugs»), etc. 


\section{R. CURTET et K. DJONIN}

\section{b) Comparaison a nos essais.}

Les figures $25 a$ et $b$ pour le dégazage et $26 a$ et $b$ pour le transport montrent nos résultats d'essais exprimés dans le diagramme de Wallis et comparés à la courbe proposée par cet auteur [(19 ou 20), $k=1]$.

On peut constater que les points relatifs au dégazage se trouvent dans une plage qui entoure la courbe de Wallis. Par contre, pour le transport, les résultats expérimentaux sont parfois éloignés de cette courbe; certains points sont même (fig. 26 b) au-dessous de l'axe des abscisses et correspondent à des valeurs négatives de $V_{12}$, ce qui est physiquement impossible d'après le modèle de Wallis.

\section{D - Schéma de Zuber-Findlay [6].}

Zuber et Findlay basent leurs calculs sur les mêmes hypothèses que celles de Wallis, avec cette seule différence que les profils de vitesses et de concentration ne sont pas uniformes. Soit $v_{y}$ la vitesse de glissement locale, différence entre la vitesse vraie de la phase dispersée $v_{2}$ et la vitesse vraie de la phase continue $v_{1}$ :

$$
v_{g}=v_{2}-v_{1}
$$

Soient $j_{1}$ et $j_{2}$ les vitesses apparentes :

$$
j_{1}=(1-c) v_{1} \quad \text { et } \quad j_{2}=c v_{2}
$$

$j$ la vitesse locale de l'émulsion :

$$
j=j_{1}+j_{2}
$$

Les auteurs appellent « vitesse de dérive » (drift velocity) le terme $V_{2 j}$ tel que :

$$
\mathrm{V}_{2 j}=v_{2}-j
$$

Une fonction $F$ donnée a pour valeur moyenne dans la section $\mathrm{S}$ :

$$
<\mathrm{F}>=\frac{1}{\mathrm{~S}} \iint_{\mathrm{S}} \mathrm{F} d \mathrm{~S}
$$

La moyenne pondérée $\overline{\mathrm{F}}$ est définie par la relation :

$$
\overline{\mathbf{F}}=\frac{\langle\mathrm{F} c\rangle}{\langle c\rangle}
$$

a) Diagramme de Zuber-Findlay :

Si $Q_{1}$ et $Q_{2}$ sont les débits totaux de phase continue ef de phase dispersée :

$$
<j_{1}>=<(1-c) v_{1}>=\frac{\mathrm{Q}_{1}}{\mathrm{~S}}
$$

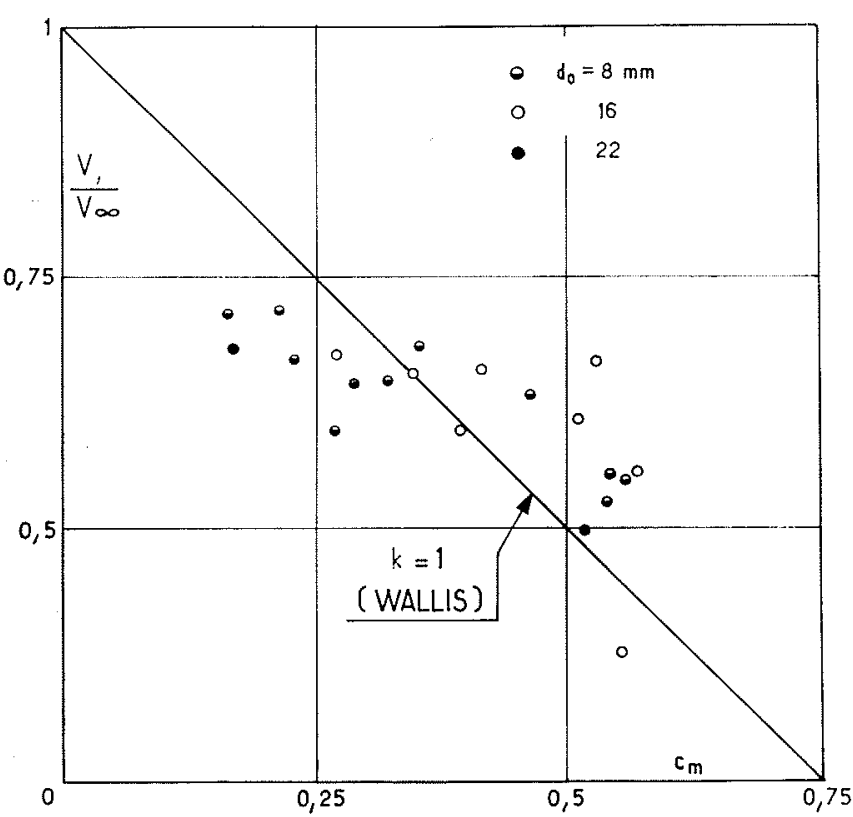

(a)

DEGAZAGE

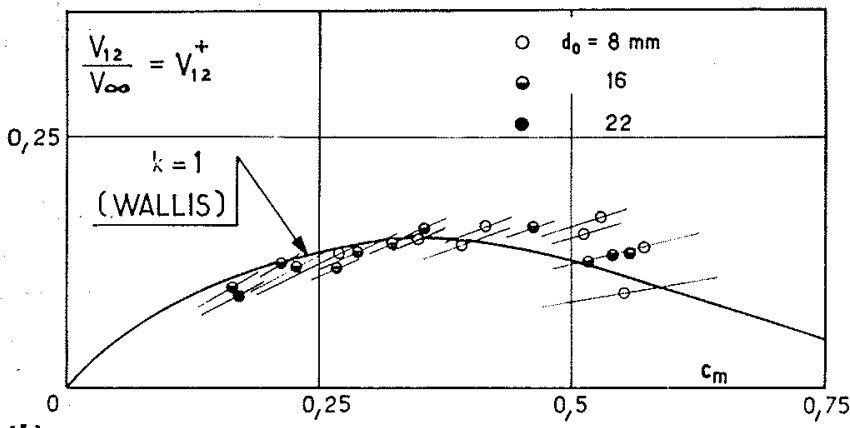

(b)

25 a et b/ Dégazage.

No air entrainment case.

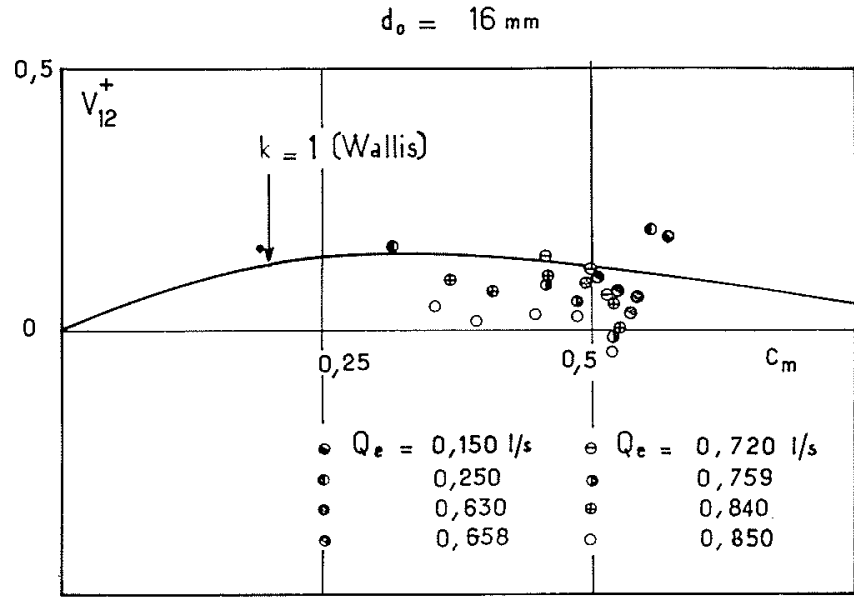

(a)

$d_{0}=22 \mathrm{~mm}$

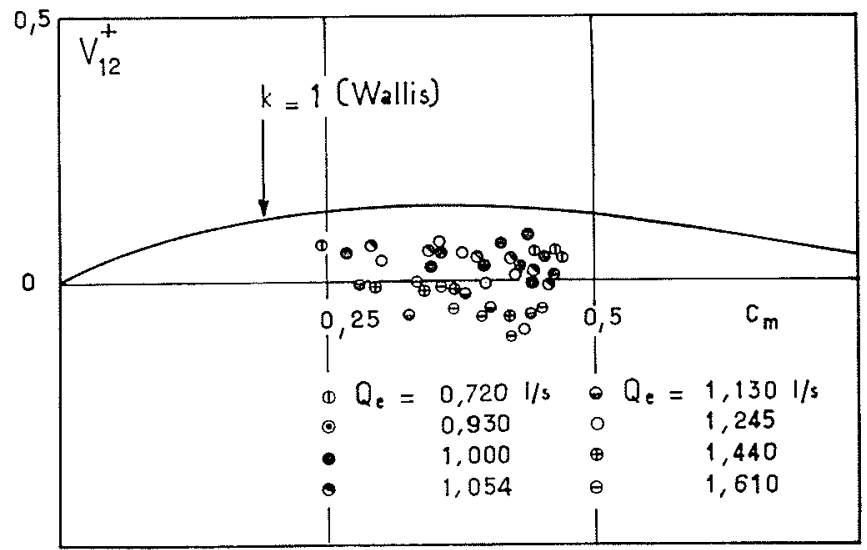

(b)

26 a ef b/ Transport. Entrainment. 


$$
\begin{gathered}
<j_{2}>=<c v_{2}>=\frac{\mathrm{Q}_{2}}{\mathrm{~S}} \\
<j>=<j_{1}>+<j_{2}>=\frac{\mathrm{Q}_{1}+\mathrm{Q}_{2}}{\mathrm{~S}}
\end{gathered}
$$

D'après (27) et (29) :

$$
\bar{v}_{2}=\frac{\left\langle v_{2} c\right\rangle}{\langle c\rangle}=\frac{\left\langle j_{2}\right\rangle}{\langle c\rangle}=\frac{Q_{2}}{\mathrm{~S}\langle c\rangle}
$$

La moyenne de (25) multipliée au préalable par $c$ conduit à la relation :

$$
<v_{2} c>=\left\langle c \mathrm{~V}_{2 j}>+<j c\right\rangle
$$

qui peut s'écrire :

$$
\left.\frac{\mathrm{Q}_{2}}{\mathrm{~S}\langle c\rangle}=\frac{\left\langle c \mathrm{~V}_{2 j}\right\rangle}{\langle c\rangle}+\frac{\langle j c\rangle}{\langle c\rangle\langle j\rangle}<j\right\rangle
$$

oul :

avec :

$$
\frac{\mathrm{Q}_{2}}{\mathrm{~S}<c>\mathrm{V}_{\infty}}=\mathrm{C}_{0}\left(\frac{\mathrm{Q}_{1}+\mathrm{Q}_{2}}{\mathrm{SV}_{\infty}}\right)+\mathrm{C}_{1}
$$

$$
\mathrm{C}_{0}=\frac{\langle j c\rangle}{\langle c\rangle\langle j\rangle}
$$

et :

$$
\mathrm{C}_{1}=\frac{\left\langle c \mathrm{~V}_{2 j}\right\rangle}{\langle c\rangle \mathrm{V}_{\infty}}
$$

Zuber et Findlay admettent que pour une émulsion donnée, $\mathrm{C}_{0}$ et $\mathrm{C}_{1}$ sont des constantes, de sorte que l'équation (34) se traduit par une droite dans le diagramme proposé par les auteurs (fig. 27). En abscisses, est porté le débit de l'émulsion exprimé sous forme adimensionnelle; en ordonnées, le terme $\mathrm{Q}_{2} / \mathrm{S}\langle c\rangle \mathrm{V}_{\infty}$, vitesse moyenne caractéristique de la phase dispersée. Notons que, pour nos essais, $Q_{1}=Q_{e}, Q_{2}=Q_{a},\langle c\rangle=c_{m}$.

\section{b) Calcul des coefficients $\mathrm{C}_{0}$ et $\mathrm{C}_{1}$ :}

Les auteurs proposent de représenter les profils de vitesse $j$ et de concentration $c$ par les expressions :

et :

$$
\frac{j}{j_{c}}=1-\left(\frac{r}{\mathrm{R}}\right)^{m}
$$

$$
\frac{c}{c_{c}}=1-\left(\frac{r}{\mathrm{R}}\right)^{n}
$$

$j_{c}$ et $c_{c}$ étant les valeurs de $j$ et de $c$ sur l'axe, $\mathrm{R}$ le rayon de la conduite. Compte tenu de ces équations, la relation (35) conduit à :

$$
\mathrm{C}_{0}=\frac{m+n+4}{m+n+2} \text { et, si } m=n, \mathrm{C}_{0}=\frac{n+2}{n+1}
$$

Pour $m=n=10, \mathrm{C}_{0}=1,09$ et si $m=n=20$, $\mathrm{C}_{0}=1,05$. On voit que la pente $\mathrm{C}_{0}$ de la droite est proche de 1 (fig. 27). Elle est égale à 1 si la répartition des vitesses et des concentrations est uniforme.

Pour le calcul de $C_{1}$, il est nécessaire d'expliciter la vitesse de transport $\mathrm{V}_{2 j}$. Un calcul simple montre que :

$$
\mathrm{V}_{2 j}=v_{g}(1-c)
$$

Zuber et Findlay admettent, comme Wallis, que la vitesse de glissement locale $v_{g}$ est de la forme:

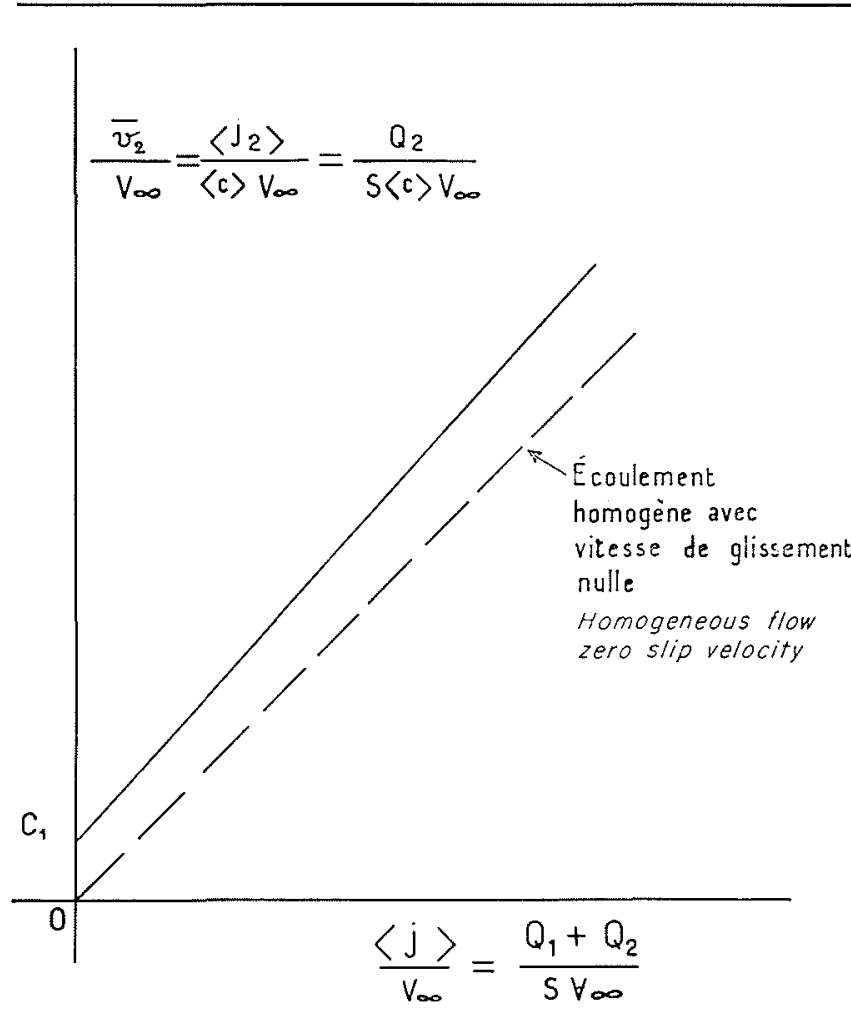

27/ Diagramme de Zuber-Findlay. Zuber-Findlay diagram.

$$
v_{g}=\mathrm{V}_{\infty}(1-c)^{k}
$$

ou, d'après (40) et (41):

$$
\mathrm{V}_{2 j}=\mathrm{V}_{\infty}(1-c)^{k+1}
$$

Le calcul du terme $\mathrm{C}_{1}$ défini par (36) conduit à :

$$
\begin{aligned}
& \mathrm{C}_{1}=1 \quad \text { si } k=0 ; \\
& \mathrm{C}_{1}=1-\frac{n c_{r}}{n+1} \text { pour } k=1 ; \\
& \mathrm{C}_{1}=1-\frac{2 n c_{r}}{n+1}-\frac{3 c_{c}^{2} n^{2}}{(n+1)(3 n+2)} \text { pour } k=2 ; \\
& \mathrm{C}_{1}=\left(1-c_{c}\right)^{k+1}, \text { si } n \text { est grand devant l'unité. }
\end{aligned}
$$

On constate que le terme $\mathrm{C}_{1}$ dépend de la concentration sur l'axe $c_{c}$ et que, en toute rigueur, il n'est pas légitime de le considérer comme une constante; toutefois, si les variations de concentration sur l'axe sont relativement peu étendues, on peut admettre comme ordonnée à l'origine de la droite de $Z$ uber-Findlay une valeur moyenne de $C_{1}$.

Par ailleurs, les auteur's conseillent d'utiliser leur diagramme en régime établi seulement, afin que les coefficients $m$ et $n$ ne varient pas d'une section à l'autre de la conduite.

\section{c) Comparaison aux mesures.}

Compte tenu des valeurs expérimentales de l'exposant $n$ (38) et de $c_{c}$, nous avons admis pour le diaphragme de $16 \mathrm{~mm}, \mathrm{C}_{0}=1,07$ et $\mathrm{C}_{1}=0,3$, et pour l'orifice de $22 \mathrm{~mm}, \mathrm{C}_{n}=1,09$ et $\mathrm{C}_{1}=0,4$. Puis nous avons tracé les deux droites correspondant à ces coefficients, et reporté les points expérimentaux 


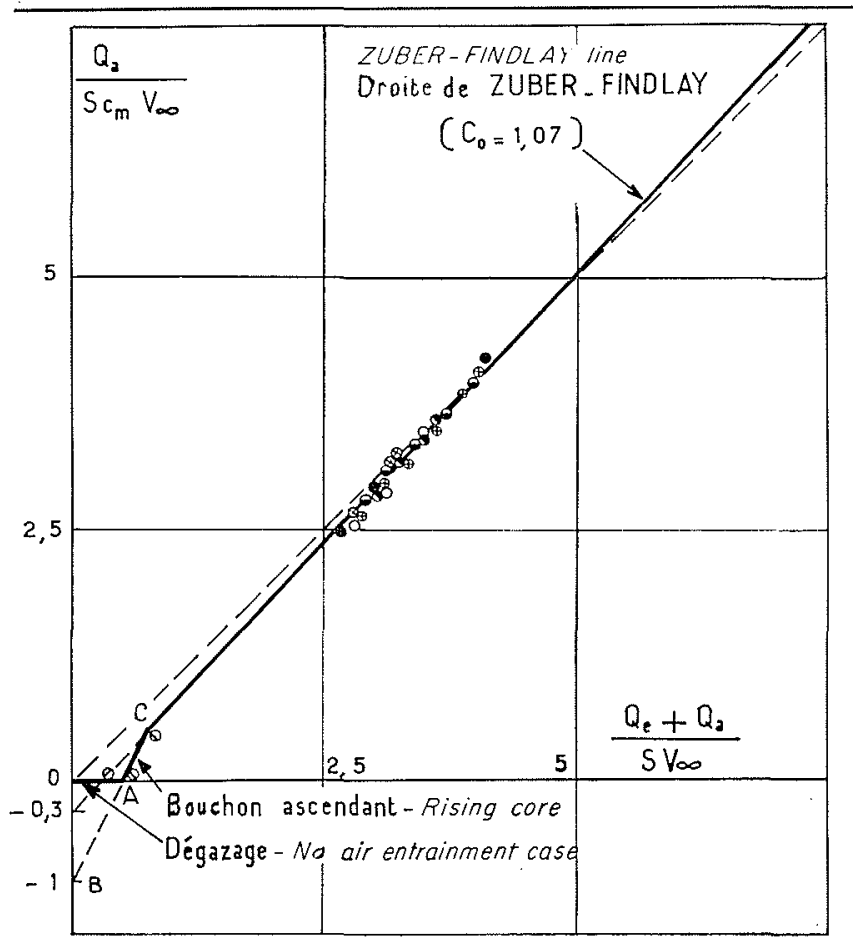

(a)

$$
\underline{\mathrm{d}_{0}=16 \mathrm{~mm}}
$$
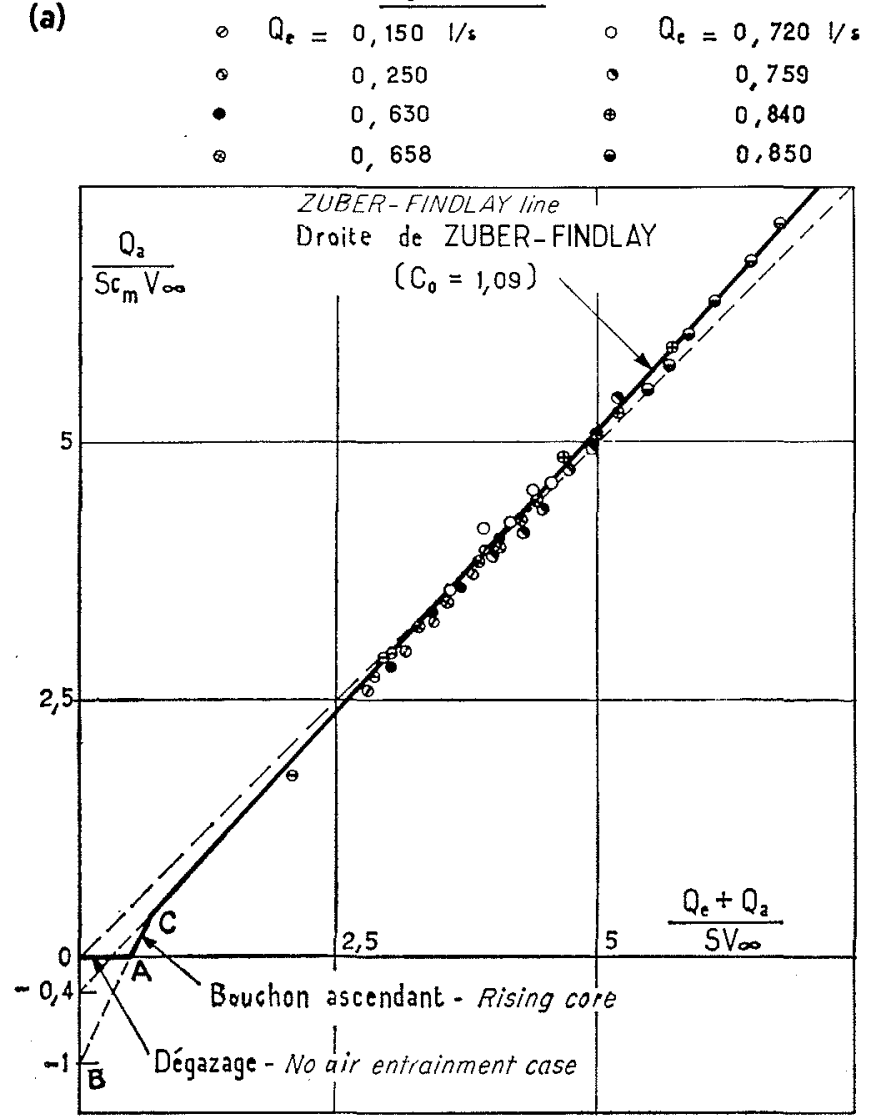

(b)

\begin{tabular}{|c|c|c|c|}
\hline \multicolumn{4}{|c|}{$d_{0}=22 \mathrm{~mm}$} \\
\hline & $Q_{e}(L / s)$ & & $Q_{e}(1 / s)$ \\
\hline$\theta$ & 0,720 & 0 & 1,130 \\
\hline 0 & 0,930 & 0 & 1,245 \\
\hline$\bullet$ & 1,000 & $\oplus$ & 1,440 \\
\hline$\otimes$ & 1,054 & $\bullet$ & 1,610 \\
\hline
\end{tabular}

28 a ef b/ Comparaison de nos résultats d'essais au schéma de Zuber-Findlay, avec $d_{0}=16 \mathrm{~mm}$ et $d_{0}=22 \mathrm{~mm}$. Comparison between the authors tests results and the Zuber-Findlay diagram, with $\mathrm{d}_{0}=16 \mathrm{~mm}$ and $\mathrm{d}_{0}=22 \mathrm{~mm}$. sur un diagramme de Zuber-Findlay : le résultat obtenu apparaît sur les figures $28 a$ et $b$.

Notons tout d'abord que l'ordonnée à l'origine des droites est négative. En effet, la vitesse $V_{2}$ est orientée vers le haut comme la vitesse de glissement $v_{g}$, alors que les débits d'eau et d'air sont dans le cas actuel orientés vers le bas : il en résulte que $\mathrm{C}_{1}$ doit avoir un signe contraire au débit d'air $\mathbf{Q}_{a}$.

On constate par ailleurs que les droites passent à travers les points de mesure, mais le fait le plus remarquable est que ces droites recoupent la bissectrice des axes, en un certain point d'intersection, défini par la condition :

$$
\frac{\mathrm{Q}_{a}}{\mathrm{~S} c_{m} \mathrm{~V}_{\infty}}=\frac{\mathrm{Q}_{e}+\mathrm{Q}_{a}}{\mathrm{~S} \mathrm{~V}_{\infty}} \quad \text { ou } \quad c_{m}=c_{t}
$$

Revenons maintenant à la définition de la vitesse moyenne de glissement $V_{g}$ vue antérieurement (chap. III) :

$$
\mathrm{V}_{g}=\frac{\mathrm{Q}_{a}}{\mathrm{~S} c_{m}}-\frac{\mathrm{Q}_{e}}{\mathrm{~S}\left(1-c_{m}\right)}
$$

Cette équation peut s'écrire :

$$
\frac{\mathrm{SV}_{a}}{\mathrm{Q}_{a}+\mathrm{Q}_{c}} c_{m}\left(1-c_{m}\right)=c_{t}-c_{m}
$$

On voit que $V_{g}$ a le signe de $c_{t}-c_{m}$ et s'annule si $c_{m}=c_{t}$.

Si nous nous reportons aux figures $28 a$ et $b$, pour les débits relatifs d'émulsion inférieurs à 4 environ (point d'intersection), la vitesse $V_{g}$ est négative, et positive au-delà de 4; autrement dit, l'air paraît en moyenne s'écouler plus vite que l'eau, si le débit d'émulsion est élevé. Nous retrouvons un résultat déjà noté antérieurement, mais qui présente le grand intérêt d'être non seulement expérimental, mais justifié par le raisonnement.

Il reste à expliquer la contradiction apparente entre le fait que les vitesses de glissement locales

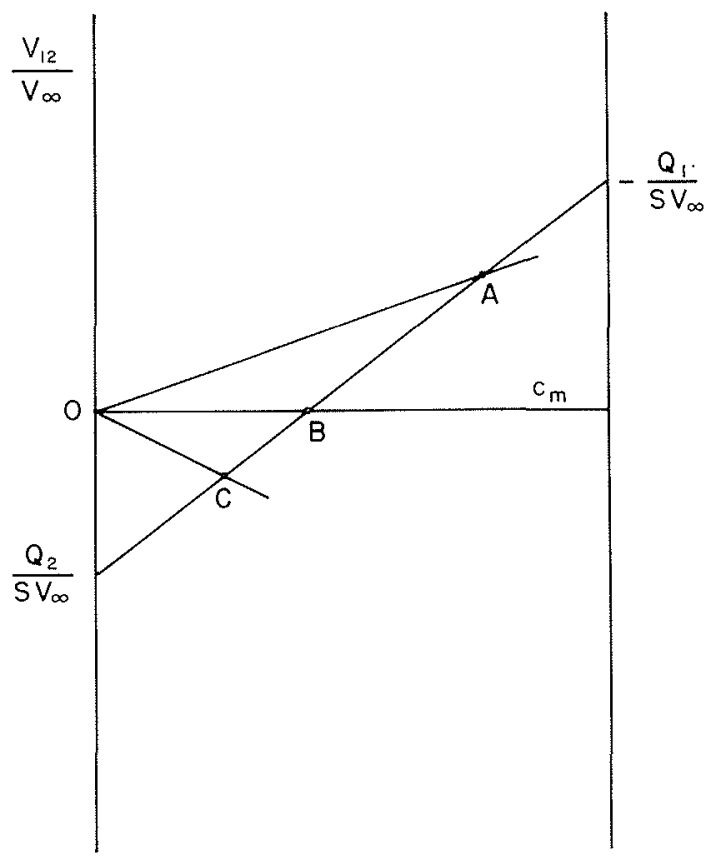

29/ Diagramme de Wallis généralisé. Generalised Wallis diagram. 
sont toutes orientées vers le haut, alors que la vitesse $V_{g}$ peut ètre dirigée selon les cas soit vers le haut, soit vers le bas. L'explication est très simple : la moyenne des vitesses de glissement s'écrit :

$\left\langle v_{g}\right\rangle=\left\langle v_{2}\right\rangle-\left\langle v_{1}\right\rangle=\left\langle\frac{j_{2}}{c}\right\rangle-\left\langle\frac{\underline{j_{1}}}{1-c}\right\rangle$

La comparaison des formules (44) et (46) montre que la vitesse $V_{g}$ n'est pas en général la moyenne des vitesses de glissement : elle ne l'est que dans le cas particulier d'une répartition uniforme des débits et des concentrations dans la section.

\section{d) Remarques.}

$1^{\circ}$ Sur les figures $28 a$ et $b$, nous avons porté le point $A$ correspondant à $Q_{a}=0$ et à $Q_{e}=0,22 \mathrm{l} / \mathrm{s}$ : le segment $\mathrm{OA}$ est relatif au dégazage. La portion de droite AC, définie par Zuber et Findlay, pourrait traduire l'apparition de poches d'air : effectivement, nos points expérimentaux se placent dans cette région.

$2^{\circ}$ La droite obtenue par ces auteurs offre l'avantage de montrer qu'il existe une relation entre les débits d'eau et d'air et la concentration moyenne : elle permet ainsi de répondre à la question posée au début de l'étude des concentrations.

$3^{\circ}$ Pour le dégazage, il semble que l'on puisse admettre, au moins en première approximation, le schéma de Wallis, lequel fournit dans ce cas particulier une relation entre la concentration moyenne et le débit d'eau. On doit noter à ce propos que les points portés sur les figures 25 ne concernent que des longueurs d'émulsion supérieures à $40 \mathrm{~cm}$.

\section{e) Généralisation du diagramme de Wallis.}

Comme nous allons le montrer à l'aide du calcul élémentaire qui suit, il est possible de reporter les résultats obtenus par Zuber et Findlay (fig. 27) dans un diagramme analogue à celui de Wallis (fig. 24), mais plus général.

Considérons une vitesse moyenne caractéristique de l'émulsion définie, pour une distribution non uniforme de concentration, par la relation :

$$
\mathrm{V}_{12}=\mathrm{V}_{g} c_{m}\left(1-c_{m}\right)
$$

Compte tenu de l'équation (44), on obtient, après division par $\mathrm{V}_{\infty}$ :

$$
\frac{\mathrm{V}_{12}}{\mathrm{~V}_{\infty}}=\frac{\mathrm{Q}_{2}}{\mathrm{SV}}\left(1-\mathrm{c}_{m}\right)-\frac{\mathrm{Q}_{1}}{\mathrm{SV}} c_{\infty}
$$

Dans un système de coordonnées comportant la concentration moyenne $c_{m}$ en abscisses et le rapport des vitesses $V_{12} / V_{\infty}$ en ordonnées (fig. 29), la relalion (48) se traduit par une droite passant par les points $\left(0, \mathrm{Q}_{2} / \mathrm{SV} \mathrm{N}_{\infty}\right)$ et $\left(1-\mathrm{Q}_{1} / \mathrm{SV} \mathrm{V}_{\infty}\right) \mathrm{Q}_{1}$ et $\mathrm{Q}_{2}$ étant respectivement les débits des phases continue et discontinue.

Les calculs de Zuber et Findlay conduisent, comme on l'a vu précédemment, à l'équation :

$$
\frac{\mathrm{Q}_{2}}{\mathrm{SV}_{\infty} c_{m}}=\mathrm{C}_{0}\left(\frac{\mathrm{Q}_{1}+\mathrm{Q}_{2}}{\mathrm{SV}_{\infty}}\right)+\mathrm{C}_{1}
$$

Posons $\mathrm{C}_{0}=1+\mathrm{C}_{2}$; l'équation (34) peut s'écrire, d'après l'expression (48), sous la forme :

$$
\frac{V_{12}}{V_{\infty}}=\left[\mathrm{C}_{2}\left(\frac{\mathrm{Q}_{1}+\mathrm{Q}_{2}}{\mathrm{~S} \mathrm{~V}_{\infty}}\right)+\mathrm{C}_{1}\right] c_{m}
$$

Celte relation se traduit sur la figure 29 par une droite passant par l'origine. Selon les valeurs des débits, la pente de cette droite peut être positive $(\mathrm{OA})$ ou négative $(\mathrm{OC})$; elle est nulle dans le cas particulier $(\mathrm{OB})$ où :

$$
\frac{\mathrm{Q}_{1}+\mathrm{Q}_{2}}{\mathrm{SV} \mathrm{V}_{\infty}}=-\frac{\mathrm{C}_{1}}{\mathrm{C}_{2}}
$$

Chaque point de fonctionnement est déterminé par l'intersection de deux droites : une telle construction permettrait dans le cas actuel de retrouver sensiblement les résultats obtenus sur les figures 26 . Il est remarquable de constater que cette construction est plus simple dans le cas général que dans le cas particulier du régime uniforme traité par Wallis.

\section{VI. - Conclusions}

L'analyse de l'écoulement biphasé que nous venons d'étudier a nécessité trois séries de recherches : tout d'abord, examen des conditions générales, régies par les équations de Bernoulli et d'Euler; puis entraînement par le jet, dont les effets indirects peuvent être connus grâce à des formules empiriques; enfin lois d'équilibre de l'émulsion, où se retrouve un bon accord entre l'expérience et les schémas classiques.

L'ensemble de ces résultats permet de résoudre un problème particulier posé : en effet, pour le transport par exemple, si l'on se donne le diaphragme, le débit d'eau et la longueur de chute libre, sur une installation donnée, toutes les autres variables peuvent en être déduites, et notamment le débit d'air et la concentration moyenne.

Parmi les questions que nous aimerions préciser dans la suite, se trouve la notion de régime établi dans l'émulsion, mais cette étude nécessite la construction d'un ensemble de mesure pouvant être déplacé le long de la conduite.

Bibliographie

[1] DJonin (K.). - Etucle de l'entrainement d'air par un jet d'eau dans une conduite verticale, Thèse de DocteurIngénieur, Faculté des Sciences de Grenoble (mai 1966).

[2] Mondin (H.). - L'ébullition et le transfert de chaleur, $7^{\circ}$ Journées de l'Hydraulique de la S.H.F., Question II, Rapport Général, Paris (1962).

[3! Dumitrescu (D.T.). - Strömung an einer Luftblase im senkrechten Rohr, Zeit. angew. Math. Mech., $\mathrm{n}^{\circ} 3$ (juin 1943), p. 139-149.

[4] Davies (R.M.) et TaYlon (Sir Geoffrey). - The mechanics of large bubbles rising through extended liquids and through liquids in tubes, Proc. Roy. Society, Londres (1950), vol, 200, séries $A$, p. 375-390.

[5] WALLIS (G.B.). - Two-phase flow phenomena associated with the boiling of liquids, Ph.D. Thesis, Cambridge University (G.-B.), (juin 1961).

[6] Zuber (N.) et Findlay (J.A.). - Average volumetric concentration in two-phase flow systems, Transactions A.S.M.E., Journal of Heat Transfer (novembre 1965), p. $453-468$. 


\section{Abstract \\ Study of a vertical downward mixed water and air flow. \\ Flow and concentration conditions}

by R. Curtet * and K. Djonin * *

The authors consider the emulsion formed by a jet impinging upon a free water surface in an upright cylindrical pipe. Starting out from set initial data, they observe three main types of two-phase flow: (1) flow without air entrainment (2) practically stable air entrainment and (3) air entrainment with unstable pockets.

This paper comprises three basic parts, one for each stage to be covered in order to gain an overall view of the question:

(i) General equations based on simplifying assumptions but nevertheless closely agreeing with experimental data;

(ii) Air entrainment by the jet, expressed by empirical formulae at this stage;

(iii) The general 'mechanism' of the emulsion, which was first investigated experimentally by recording air-in-water concentration profiles by the gamma-ray method and comparing them with Wallis and Zuber-Findlay diagrams. The first of these is adequate for flow without air entrainment, and the second, which is based on non-uniform velocity and concentration distributions, enables the results of measurements to be interpreted in the entrained air case and a simple relationship to be put forward between mean concentration and the rates of air and water flow.

* Maitre de Recherches au C.N.R.S.

* Docteur-Ingćnicur, Ingénicur au Laboratoire d'Avala (Yougoslavie). 